\title{
Hydromechanical signals in the plankton
}

\author{
André W. Visser* \\ Department of Marine Ecology and Aquaculture, Danish Institute for Fisheries Research, Kavalergården 6, \\ 2920 Charlottenlund, Denmark
}

\begin{abstract}
The distance at which plankters can detect and thus interact with each other depends on their sensitivity, size, and motion, as well as the hydrodynamic characteristics of their behaviour. Through a simple consideration of the distribution of forces exerted on the ambient fluid by different plankton behaviours, it is possible to deduce the spatial scale over which the associated hydromechanical disturbance propagates. At low Reynolds numbers, for passive sinking or for a feeding current, the associated hydromechanical velocity, $u$, attenuates with distance, $r$, as $u \propto a U r^{-1}$ where $a$ is the length scale of the organism and $U$ is its velocity relative to the fluid. Similarly, for a self-propelled organism, $u \propto a^{2} U r^{-2}$. In contrast, at high Reynolds numbers, a self-propelled organism generates a forward hydromechanical disturbance that has the form $u \propto a^{3} U r^{-3}$. Within this context, observed planktonic interactions, particularly for copepods, were analysed and showed reasonably good support for the theory. The remote detection of inert particles by feeding-current-generating and free-swimming copepods was found to be feasible for known copepod sensitivities. Directional information and signal timing for flow disturbances and vortices provided a means of locating active organisms. Finally, the effect of turbulence was considered, as it can impair a copepod's detection ability. A simple analysis of ambush-feeding copepods detecting swimming ciliates under turbulent conditions showed good agreement with previously reported observations.
\end{abstract}

KEY WORDS: Zooplankton · Copepod · Motility · Detection · Hydromechanical signal · Turbulence Resale or republication not permitted without written consent of the publisher

\section{INTRODUCTION}

Many plankton species exhibit rheotactic abilities; that is, they respond behaviourally to hydromechanical signals mediated through the surrounding fluid and originating from a disturbance generated by a potential predator, prey or mate. This ability to detect and react to remote fluid disturbances appears to be well developed in many copepod species (e.g. Kerfoot 1978, Haury et al. 1980, Yen et al. 1992, and studies cited below). Similar rheotactic abilities have been observed in other planktonic organisms such as rotifers (Kirk \& Gilbert 1988, Williamson \& Vanderploeg 1988), chaetognaths (Newbury 1972, Feigenbaum \& Reeve 1977, Nishii 1998), ciliates (Jonsson \& Tiselius 1990, Jack \& Gilbert 1993, Gilbert 1994, Jakobsen 2001) and

*E-mail: awv@dfu.min.dk ctenophores (Costello et al. 1999). Questions as to how plankton perceive their environment, how they find food, and how they interact with each other, are largely related to the character and propagation of hydromechanical signals, how these are detected, and the information they carry.

Physiologically, copepods appear to be well equipped to detect hydromechanical signals. Their first antennae are covered with an array of setae, which are highly sensitive to fluid motion (e.g. Strickler \& Bal 1973, Gill 1985, Yen et al. 1992, Bundy \& Paffenhöfer 1993, Lenz et al. 1996). Sensitivity appears to be primarily associated with the velocity difference (rather than displacement or acceleration) between the tip and base of setae (Yen et al. 1992). For instance, neurological activity can be initiated by a relative fluid velocity as low as $20 \mu \mathrm{m} \mathrm{s}^{-1}$ (Yen et al. 1992). Furthermore, the sensitivity of copepods to fluid disturbance can be deduced from a wide variety of behavioural 
studies. Chief amongst these is the escape reaction many copepods exhibit in response to the remote detection of a potential predator. Such studies include artificially generated fluid disturbances, e.g. siphon flows (Singarajah 1969, Fields \& Yen 1997b, Viitasalo et al. 1998, Kiørboe et al. 1999), translating bodies (Haury et al. 1980), oscillating bodies (Lenz \& Hartline 1999), oscillating chambers, couette shear and fluid rotation (Kiørboe et al. 1999), as well as natural fluid disturbances, e.g. copepod feeding currents (Fields \& Yen 1997a) and an approaching fish larva (Viitasalo et al. 1998). Other behavioural studies also suggest a response to hydromechanical signals. For instance, Doall et al. 1998) observed male copepods homing in on the swimming signal of females in the last phase of mate pursuit. Likewise, van Duren et al. (1998) suggested that female Temora longicornis advertise their position to potential mates through hydromechanical signals. Similarly, Svensen \& Kiørboe 2000) reported the remote detection of swimming dinoflagellates by an ambush-feeding cyclopoid copepod, and Williamson (1987) and Williamson \& Vanderploeg (1988) reported observations of remote detection of rotifers by diaptomid copepods and vice versa. Copepods are also able to detect and react to turbulence, and change their behaviour accordingly (Costello et al. 1990, Marrasé et al. 1990, Caparroy et al. 1998, Hwang et al. 2000). Escape jumps triggered by the detection of a hydromechanical signal can themselves alert a predatory copepod to the presence of a prey and initiate an attack (Williamson \& Vanderploeg 1988, Yen \& Strickler 1996). Finally, there is some evidence to suggest that copepods can remotely detect inert particles (i.e. non-motile and chemically neutral) entrained into their feeding current (Buskey 1984, Gill \& Poulet 1988, Vanderploeg et al. 1990) or approached by a free-swimming copepod (Bundy et al. 1998).

In contrast to neurological sensitivity, the threshold velocity required to elicit a behavioural response is typically higher. For instance, in a series of experiments, Kiørboe et al. (1999) show that the escape reaction of Acartia tonsa females (equivalent spherical radius $230 \mu \mathrm{m}$ ) is consistently initiated when the deformation-induced velocity difference at their antennular tips is $130 \pm 30 \mu \mathrm{m} \mathrm{s}^{-1}$. This is comparable to direct measurements of 'rapid swim' reactions elicited in a calanoid copepod, Undinula vulgaris, at 36 to $86 \mu \mathrm{m} \mathrm{s}^{-1}$ (Lenz \& Hartline 1999). A summary of escape experiments (Kiørboe et al. 1999) indicates that the escape threshold signal varies considerably, and is often much higher $(10$ to $100 \times)$ than those quoted here. The threshold velocity required to elicit an attack response has been less well documented, although Kiørboe et al. (1999) deduced that the threshold sensitivity of Oithona similis in attacking motile prey is of the order of $40 \mu \mathrm{m} \mathrm{s}^{-1}$. Given the current understanding of copepod sensitivity, I assume throughout this work, that the primary stimulus eliciting a response in a copepod is a velocity difference between its solid body and a seta positioned along its antennae. The detection mechanism for other zooplankton species such as ciliates is different, and may depend on other characteristics of the flow field. Furthermore, different setae positioned along copepods' antennae appear to be sensitive to different components of velocity disturbance (Yen et al. 1992). While I will briefly consider this aspect, I will in general assume that detection is independent of directional information of the flow perturbation.

How an organism moves through water depends on the forces that act on it. Primarily these are buoyancy, thrust and drag. Of these, thrust and drag act not only on the organism, but also on the fluid, producing hydromechanical disturbances that spread out from the moving organism. For those unfamiliar with hydrodynamics, an excellent starting point for understanding the fluid physics relevant for plankton can be found in both Denny (1993) and Vogel (1994). In general, the strength of the hydromechanical disturbance depends on the size and speed of the moving organism. In this work I will try to promote 2 ideas: firstly that the spatial pattern of the hydromechanical signal generated by a moving organism is different for different classes of motility (e.g. passive sinking, uniform swimming, rapid jump); secondly that the spatial scale, together with a plankter's sensitivity, can be used to determine its reaction distance to other organisms with relative motion (e.g. potential prey, mate or predator). In this way, I seek to quantify the potential transfer of information to and from moving organisms disturbing their fluid environment.

The dependence of reaction distance on an organism's motile characteristics has direct implications on encounter rate. Following Evans (1989), the rate, $Z_{\mathrm{pq}}$, at which a plankter $\mathrm{p}$ reacts to a planktonic prey (or mate, or predator) $\mathrm{q}$ with concentration $c_{\mathrm{q}}$ is given by

$$
Z_{\mathrm{pq}}=c_{\mathrm{q}} \pi R_{\mathrm{pq}}^{2}\left(u_{\mathrm{p}}^{2}+u_{\mathrm{q}}^{2}+2 w^{2}\right)^{1 / 2}
$$

where $u_{\mathrm{p}}$ and $u_{\mathrm{q}}$ are the swimming velocity of $p$ and $q$ respectively, $w$ is the turbulent velocity scale, and $R_{\mathrm{pq}}$ is the reception distance of $\mathrm{p}$ on $\mathrm{q}$. However, $R_{\mathrm{pq}}$ is not invariant, but depends, amongst other things, on the behavioural aspects of the encounter. In general, this can be written as $R_{\mathrm{pq}}\left(s_{\mathrm{p}}, u_{\mathrm{p}}, u_{\mathrm{q}}, w\right)$. That is, the reaction distance is a function of the plankter's sensitivity $s_{\mathrm{p}}$, its own motion as well as that of its encounter partner $\mathrm{q}$, and the turbulent velocity $w$. While some of these functional dependencies are not immediately obvious, I will try to demonstrate their influence in the sections that follow. Thus, all other things being equal, a plankter's encounter rate with a motile prey is higher 
than that with a passive prey because of 2 factors: firstly, through the increased relative motion, and secondly because a motile prey is hydromechanically more conspicuous, leading to a greater reaction radius $R_{\mathrm{pq}}$.

The specific hydromechanical disturbance generated by a plankter depends on the sum of the forces it exerts on the fluid, their spatial distribution, the boundary conditions imposed by the solid body of the plankter, and the Reynolds number regime in which the flow is generated. This latter is formally defined as: $R e=\rho L U / \mu$, where $\rho$ is the density of the fluid, $L$ is the length scale of the body, $U$ is its velocity relative to the fluid, and $\mu$ is the dynamic viscosity of the fluid (e.g. Denny 1993, Vogel 1994). This gives the ratio of the inertial to viscous forces operating on the fluid, and it is noteworthy that $R e$ covers many decades (e.g. Yen 2000) - from $O\left(10^{3}\right)$ for a copepod escape jump (inertia dominates), to $O\left(10^{-3}\right)$ for a phytoplankton cell imbedded in a copepod feeding current where viscosity dominates. This large range in $R e$ means that different dynamical descriptions must be considered. The strategy of the present study was to examine the dynamics at the 2 extremes, for $R e \rightarrow 0$ (viscous), and for $R e \rightarrow \infty$ (inertial). These are known as the Stokes and Eulerian regimes respectively.

The first limit concerns Stokes (or creeping) flow, for which exact analytical solutions can often be found for flow induced by simple force distributions and body shapes. Furthermore, because the dynamics governing Stokes flow are linear, methods can be easily adapted for more complex systems by adding solutions together. With regard to the hydrodynamics relevant for zooplankton, this principal has been applied in both analytic (Childress et al. 1987) and computer (Jiang et al. 1999) models. Formally, however, Stokes flow is valid only for very low Reynolds number. It is somewhat surprising then, that for 3-dimensional bodies, Stokes flow solutions compare favourably, at least in bulk characteristics such as drag, with observations up to $R e=1$, with appreciable deviations only appearing at $R e>10$ (Batchelor 1967, Vogel 1994). Furthermore, where more exact solutions at finite $R e$ have been found, the Stokes flow solution generally appears as a first-order approximation.

At the other extreme $(\operatorname{Re}>10)$, where the process is inertial in character, the laminar boundary layer theory can be applied. This is widely used in aerodynamics, and assumes that viscosity is only important in a thin laminar layer immediately adjacent to a translating body. Beyond this, the flow is inertial, and is governed only by continuity and the conservation of momentum. This type of flow has a strong fore-aft asymmetry. Momentum and vorticity generated in the laminar boundary layer is shed downstream of the moving body, and the wake becomes turbulent at about $R e>100$. In front of the moving body, however, the flow is undisturbed and remains laminar at these and even higher Reynolds numbers. This can be seen in photos of the flow upstream of a sphere, as shown by Batchelor 1967: his Fig. 4. 12. 1). In several instances, it is the characteristics of the upstream flow that is important for plankton interactions.

The flow field associated with any translating 3D body will always have characteristics of both the Stokes and Eulerian regimes. Sufficiently close to the body viscosity will dominate, and sufficiently far away viscosity becomes less important and inertia tends to dominate. The distance at which this transition takes place is the boundary layer thickness: $\delta=L R e^{-1 / 2}$. As a rule of thumb, for reaction events outside of the wake, a comparison of the reaction distance, $R$, with the boundary layer thickness gives a good indication of the relevant regime: $R<\delta$ for Stokes regime and $R>\delta$ for Eulerian regime.

This work is primarily concerned with the scale of the hydromechanical signal generated by different motile behaviours of plankton. Specifically, given known mechano-sensitivities for either neurological or behavioural responses, the feasibility of mechanoreception as a means of remote detection for differing behavioural classes is explored. The specific types of behaviour and processes considered in this work were of: (1) a passive particle in a deforming flow, e.g. a phytoplankton cell entrained in a feeding current or approached by a free-swimming copepod; (2) an eccentric (i.e. bottom-heavy) particle in a rotating flow, e.g. a nauplius entrained in a feeding current; (3) a sinking particle, e.g. faecal pellet; (4) a feeding current, e.g. as produced by calanoid copepods, ciliates and bivalve larvae; (5) constant swimming at low $R e$, e.g. of ciliates, nauplii, rotifers; (6) beating of feeding appendages; (7) constant swimming at high $R e$, e.g. fish larvae; (8) a rapid jump, e.g. copepod escape reaction.

These have been arranged in approximate order of increasing Re. I approach the analysis of (1) to (5) with respect to Stokes flow, utilising the multipole expansion, an especially instructive tool for analysing such flows directly from the balance of forces and boundary conditions. The acoustics of beating appendages (6) must allow for the slight but finite compressibility of water, which imposes a speed (the speed of sound) at which pressure disturbances propagate. While it has never been demonstrated that copepods can 'hear' (i.e. are sensitive to pressure fluctuations), there are associated velocity fluctuations that can potentially be felt. Finally, a rapid jump such as in a copepod's escape response (8) is inherently an inertial process. In the simplest case, the copepod applies a single powerful 

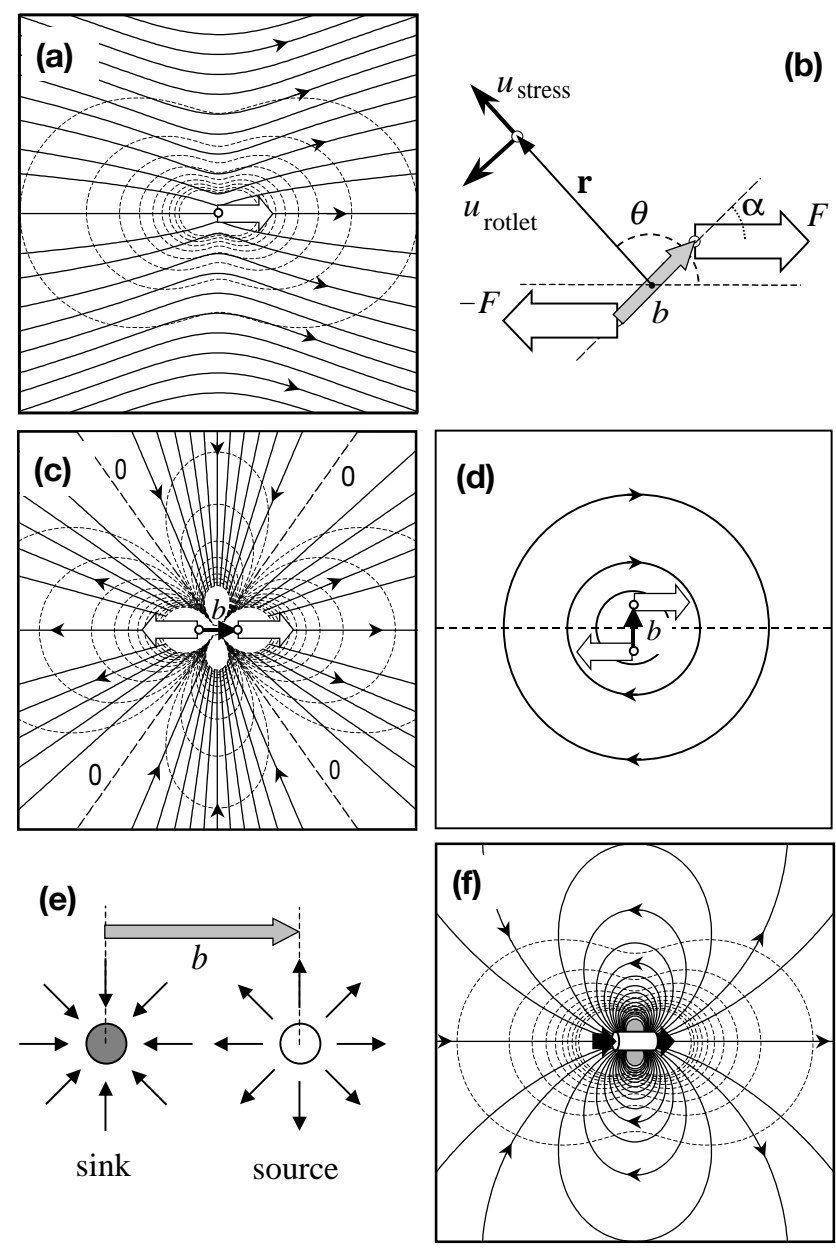

Fig. 1. Stream functions associated with (a) the stokeslet, (c) the stresslet, (d) the rotlet and (f) the potential dipole. Isotachs (lines of equal speed) are shown by dashed lines. Stream functions for the 3 cases $(\mathrm{a}, \mathrm{c}$ and $\mathrm{f})$ are given in Table 1. (b) General dipole, where forces $F$ and $-F$ are equal and opposite, and separated by the vector $b$. When $\alpha=0^{\circ}$, rotlet component disappears and the flow field corresponds to stresslet in (c); when $\alpha=90^{\circ}$, the dipole flow consists of a stresslet similar to that in (c) and a rotlet flow as in (d). Source and sink in (e) produce the potential dipole flow in (f)

impulse of momentum to the fluid, propelling itself forward and expelling a vortex in its wake. The copepod glides under its own inertia and eventually comes to rest as drag dampens its motion.

\section{STOKES FLOW SOLUTIONS}

A large class of plankton motility falls into the low Reynolds number (Stokes) regime. Indeed, the definition of plankton, being at the mercy of ambient currents, presupposes that their inherent inertia can usually be ignored. In this section we summarise a means of estimating the Stokes flow associated with force distributions. We then apply this approach to idealised cases pertinent to remote detection in the plankton.

\section{Multipole expansion}

The simplest flow field in the Stokes regime is that associated with a single force, $F$, acting on a fluid at a fixed point. The velocity field associated with this force is given by:

$$
\mathbf{u}_{\text {stokes }}(\mathbf{r}, \mathbf{F})=\frac{1}{8 \pi \mu}\left(\frac{\mathbf{F}}{r}+\frac{\mathbf{r}(\mathbf{F} \cdot \mathbf{r})}{r^{3}}\right)
$$

where $\mu$ is the dynamic viscosity, $\mathbf{r}$ is a general point in the field, and $r=|\mathbf{r}|$ is the distance from the location where force $F$ is applied (e.g. Lighthill 1975, Childress 1981, Kim \& Karrila 1991, Leal 1992). The associated stream function, $\psi_{\text {stokes }}$ (Table 1) helps to visualise this flow field (Fig. 1a). Streamlines converge towards the origin, indicating that fluid accelerates as it approaches, and decelerates as it moves away from the applied force. In addition, a broad tube of fluid is dragged parallel to the force by the effects of viscosity.

This flow field (Eq. 2) is termed a stokeslet in recognition of its fundamental nature. Because the dynamics of Stokes flows are linear, in principal any Stokes flow can be constructed by adding stokeslets together. This approach has been used in analysing for instance, flagellated swimming (Lighthill 1975, Childress 1981) and the generation of feeding currents (Childress et al. 1987, Jiang et al. 1999). For simple body shapes and force distributions, the flow field can also be modelled

Table 1. Stream functions associated with selected multipoles, expressed as spherical polar coordinates $(r, \theta, \phi ;$ Fig. 2$)$. Stream function is mathematical description of stream lines which trace the path of fluid fluid in a laminar flow. The selected flow fields are axially symmetric with respect to the azimuthal coordinated $\phi$. The components of the associated velocity field are $u_{r}$ in the radial direction and $u_{\theta}$ tangental direction (i.e. parallel to the unit vectors $\hat{r}$ and $\hat{\theta}$, respectively, in Fig. 2). See 'Stokes flow solutions', 1st subsection, for explanation of terms

\begin{tabular}{|c|c|}
\hline Term & Definition \\
\hline$\psi_{\text {stokes }}=3 / 4 \operatorname{Uar} \sin ^{2} \theta$ & Stokeslet \\
\hline$\psi_{\text {stress }}=-3 / 2 U a^{2} \cos \theta \sin ^{2} \theta$ & Stresslet \\
\hline$\psi_{\text {potdip }}=-1 / 2 U a^{3} r^{-1} \sin ^{2} \theta$ & $\begin{array}{l}\text { Potential dipole } \\
\text { (force quadrapole) }\end{array}$ \\
\hline$u_{r}=\frac{1}{r^{2} \sin \theta} \frac{\partial \psi}{\partial \theta}$ & Radial velocity \\
\hline$u_{\theta}=\frac{1}{r \sin \theta} \frac{\partial \psi}{\partial r}$ & Tangential velocity \\
\hline
\end{tabular}


by a Taylor expansion of a stokeslet at the geometric centre of a body, and evaluated on its surface. This leads to the so-called multipole expansion of Stokes flows (e.g. Kim \& Karrila 1991, Leal 1992), whereby the flow is considered as the superposition of successively higher order 'poles' such that their sum conforms to the appropriate dynamics and boundary conditions. The term 'pole' is borrowed from classical electromagnetism where, for instance, the magnetic field associated with the north and south poles of a magnet is described by a magnetic (potential) dipole. In the present context, the pole hierarchy enumerates the forces acting on the fluid, i.e. the monopole arises from a single force and is equivalent to the stokeslet. Two forces gives rise to a dipole, 4 to a quadrapole and so forth. In general, the velocity field, $u_{n}$, associated with successively higher 'poles' scales as:

$$
u_{n} \approx U\left(\frac{a}{r}\right)^{n} \text { for } n \geq 1
$$

where $U$ is a velocity scale (e.g. swimming speed, feeding current velocity, settling velocity), $a$ is a length scale (e.g. size of the plankter), $r$ is radial distance from the origin of the disturbance, and $n$ corresponds to the $2^{n-1}$ pole. The fact that this expansion is valid for $n \geq 1$ reflects the physical constraint that the fluid disturbance vanishes at $r \rightarrow \infty$.

The general principal here is that if we can identify the lowest order, $n$, associated with a particular behaviour, we have come a long way in specifying the hydromechanical scale associated with this behaviour. This is because higher-order poles, irrespective of how they are distributed, attenuate more rapidly with distance from the source.

The second-order multipole is the force dipole. Physically this represents the coupled action of 2 forces of equal and opposite strength (i.e. $\mathbf{F}$ and $-\mathbf{F}$ ), separated by the vector $\mathbf{b}$ (Fig. 1b).

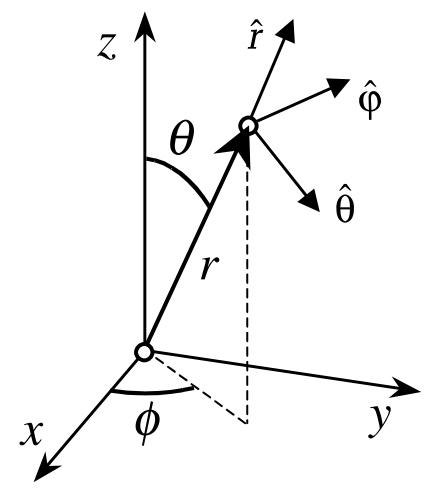

Fig. 2. Spherical polar co-ordinates $(r, \theta, \phi)$ in relationship to Cartesian coordinates $(x, y, z)$. Unit vectors $\hat{r}, \hat{\theta}$ and $\hat{\phi}$ point in $r, \theta$ and $\phi$ directions respectively
The flow thus generated is defined by:

$$
\mathbf{u}_{\mathrm{S} 2}=(\mathbf{b} \cdot \nabla) u_{\text {stokes }}(\mathbf{r}, \mathbf{F})
$$

where $\nabla$ is the divergence operator; its specific form for spherical polar co-ordinates can be found in Appendix 2 of Bachelor (1967). The general dipole can be best described as the sum of 2 components. These are the stresslet:

$$
\mathbf{u}_{\text {stress }}(\mathbf{r}, \mathbf{F}, \mathbf{b})=\frac{1}{8 \pi \mu}\left(-\frac{\mathbf{F} \cdot \mathbf{b}}{r^{3}}+\frac{3(\mathbf{F} \cdot \mathbf{r})(\mathbf{b} \cdot \mathbf{r})}{r^{5}}\right) \mathbf{r}
$$

and the rotlet:

$$
\mathbf{u}_{\text {rotlet }}(\mathbf{r}, \mathbf{F}, \mathbf{b})=\frac{1}{8 \pi \mu}\left(\frac{(\mathbf{b} \times \mathbf{F}) \times \mathbf{r}}{r^{3}}\right)
$$

The flow associated with the general force dipole is the sum of these, i.e. $\mathbf{u}_{\mathrm{S} 2}=\mathbf{u}_{\text {stress }}+\mathbf{u}_{\text {rotlet }}$. For the stresslet (Eq. 4), the flow is always radial, directed towards or away from the origin (the centre of $\mathbf{b}$ : Fig. 1b). Conversely, the rotlet flow (Eq. 5) is always directed perpendicular to the radial direction and circulates around the origin. The nature of the flow can best be seen for the 2 cases where $\mathbf{F}$ and $\mathbf{b}$ are parallel, and where $\mathbf{F}$ and $\mathbf{b}$ are perpendicular. In the first case ( $\mathbf{F}$ and $\mathbf{b}$ parallel, $\alpha=0^{\circ}$ in Fig. $1 \mathrm{~b}$ ), $\mathbf{u}_{\text {rotlet }}=0$, and $\mathbf{u}_{\text {stress }}$ is a deformation flow, given in spherical polar co-ordinates (Fig. 2) by:

$$
\mathbf{u}_{\text {stress }}(\mathbf{r}, \mathbf{F}, \mathbf{b})=\frac{|\mathbf{F} \| \mathbf{b}|}{8 \pi \mu r^{2}}\left(3 \cos ^{2} \theta-1\right) \hat{r}
$$

where $\hat{\boldsymbol{r}}$ is the unit vector in the radial direction. The associated stream function $\psi_{\text {stress }}$ (Table 1) is shown in Fig. 1c. The stresslet fields considered in this work are primarily of this form, where the force and displacement vectors are parallel. In the second case $(\mathbf{F}$ and $\mathbf{b}$ perpendicular, $\alpha=90^{\circ}$ in Fig. $1 \mathrm{~b}$ ), $\mathbf{u}_{\text {stress }}$ is again a deformation flow (similar to Fig. 1c but rotated by $45^{\circ}$ ) and $\mathbf{u}_{\text {rotlet }}$ is a pure rotational flow (Fig. 1d).

One final multipole of relevance to this work is the potential (or source) dipole with flow given by:

$$
\mathbf{u}_{\text {potdip }}(\mathbf{r}, \mathbf{Q}, \mathbf{b})=\frac{Q}{4 \pi}\left(-\frac{\mathbf{b}}{r^{3}}+\frac{3 \mathbf{r}(\mathbf{b} \cdot \mathbf{r})}{r^{5}}\right)
$$

The associated stream function $\psi_{\text {potdip }}$ is listed in Table 1. Physically, this can be seen as the flow produced by a fluid source of strength $Q$ (a volume flux, units $\mathrm{m}^{3} \mathrm{~s}^{-1}$ ) and a fluid sink of equal strength, i.e. $-Q$, separated by the vector $\mathbf{b}$ (Fig. 1e). Indeed, one can imagine this as a short section of pipe, length $|\mathbf{b}|$, sucking water in at one end at a rate, $Q$, and expelling it at the other end. This analogy however is limited in that the inertia of fluid that is expelled is not simply in the direction of the pipe, but is radially symmetric, like an inverted siphon. The stream function associated with the potential dipole is plotted in Fig. 1f. An important 
Table 2. Lowest-order property of multipole entities and their related scaling. Here, $a$ is the length scale (radial dimension) of the body in motion, $r$ the radial distance, and $U$ and $\Omega$ the velocity and rotation rate of the body with repect to the fluid, respectively

\begin{tabular}{|llc|}
\hline Property & Multipole & Velocity scaling \\
\hline Net force & Stokeslet & $\frac{a U}{r}$ \\
No net force & Stresslet & $\frac{a^{2} U}{r^{2}}$ \\
Net torque & Rotlet & $\frac{a^{3} \Omega}{r^{2}}$ \\
Zero vorticity & Potential dipole & $\frac{a^{3} U}{r^{3}}$ \\
\hline
\end{tabular}

feature of the potential dipole is that it has zero vorticity everywhere. It is this feature that makes the potential dipole important not just at low $R e$, but also at high $R e$. While this flow is commonly termed the potential (or source) dipole, it is equivalent to a force quadrapole. This nomenclature can be confusing so one must try to be consistent in using the appropriate qualifier (force or source or potential) when speaking of specific dipoles.

An important point is that each of the listed multipoles (stokeslet, the 2 force dipoles stresslet and rotlet, and the force quadrapole or potential dipole) describes a unique lowest-order property of the net force distribution or flow field. Specifically, the stokeslet exerts a net force whereas the stresslet exerts no net force on the fluid. The rotlet on the other hand is the lowestorder multipole that exerts a net torque on the fluid, while the potential dipole generates a flow field with zero vorticity. Inversely, if 1 of these specific physical properties characterises a planktonic motility behaviour, one can deduce the lowest order flow field description associated with it. That is as summarised in Table 2, for the 4 cases of non-zero mean force, no

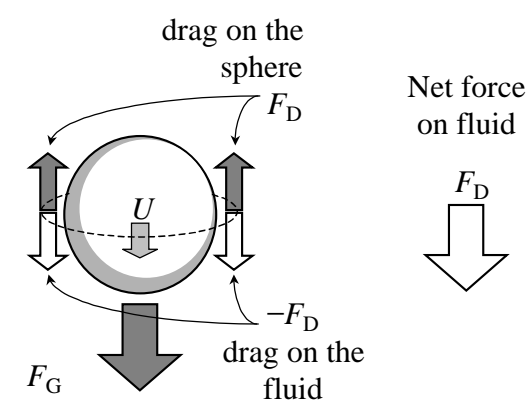

Fig. 3. Force exerted on a fluid by a settling particle. Gravity $F_{\mathrm{G}}$ and drag $F_{\mathrm{D}}$ acting on the particle balance each other in steady state. Of these, only drag acts on the fluid, so that the flow field is approximated first-order by a strokeslet to mean force, a mean torque acting on the fluid, or an irrotational flow field, the lowest order flow fields are associated with the stokeslet, the stresslet, the rotlet, and the potential dipole respectively.

\section{Passive sinking particles}

While the flow associated with a settling particle is one of the best known Stokes solutions, it serves in this case as an illustrative example from which more complex systems can be developed. A particle with density greater than its surrounding fluid reaches a steady settling velocity when the force of gravity acting on it exactly equals the drag exerted by the fluid (Fig. 3). The net force on the particle is zero. However, the net force acting on the fluid is non-zero. By Newton's second law, the net force acting on the fluid is equal and opposite to the drag exerted by the fluid on the particle. Thus, the first order flow generated by the sinking particle is a stokeslet of strength $\mathbf{F}_{\mathrm{D}}$. Stokes drag, $\mathbf{F}_{\mathrm{D}}$, is known for a variety of simple-shaped bodies (e.g. Berg 1993, Vogel 1994), For a sphere of radius $a$, sinking with a velocity $U \hat{z}$ ( $\hat{z}$ is the unit vector directed upwards), the drag exerted by the particle on the fluid is given by $\mathbf{F}_{\mathrm{D}}=-6 \pi a \mu U \hat{z}$. The second element of the flow field concerns the boundary conditions, i.e. no flow and no net stress on the body's surface. There is no systematic way of choosing the multipole distribution that, together with a specific force distribution, will ensure that boundary conditions are met; one can only use trial and error (Leal 1992). For a translating spherical body, a potential dipole acting at the geometric centre of the body is exactly the entity required. Finally, the stokeslet + potential dipole flow is relative to the far field flow. In order that the flow can be described relative to the settling body, the relative farfield motion of the fluid must be added. The final result is:

$$
\begin{array}{r}
\mathbf{u}=U\left[\begin{array}{c}
-\cos \theta \\
\sin \theta
\end{array}\right]+\frac{3}{4} \frac{a U}{r}\left[\begin{array}{c}
2 \cos \theta \\
-\sin \theta
\end{array}\right]-\frac{1}{4} \frac{a^{3} U}{r^{3}}\left[\begin{array}{c}
2 \cos \theta \\
\sin \theta
\end{array}\right] \\
\begin{array}{r}
\text { background } \\
\text { flow }
\end{array} \quad \begin{array}{r}
\text { force balance: } \\
\text { stokeslet flow }
\end{array} \quad \begin{array}{l}
\text { boundary conditions: } \\
\text { potential dipole flow }
\end{array}
\end{array}
$$

(e.g. Berg 1993). The notation used here is explained in Table 3 .

As an example, consider an ambush-feeding copepod such as Oithona similis, of antenna length $b$, feeding on settling particles of radius a sinking at speed $U$ (Fig. 4). As a sinking body falls past the copepod it will, in general, cause the copepod to move along with the ambient fluid, particularly if the particle is large compared to the copepod. The velocity of the copepod (body + antennae) induced by the settling particle is 
Table 3. Hydrodynamic flow equations associated with various biomechanical sources. All equations are written in terms of spherical polar coordinates $(r, \theta, \phi)$, where $r$ is the distance from the source of the disturbance, $\theta$ is the zenith angle, and $\phi$ the azimuthal angle (Fig. 2). Because flow fields are axially symmetric, $\theta=0$ lies parallel to the principal axis of the flow in all cases so that explicit $\phi$ dependence disappears in most cases. Flow fields are presented as vector fields with the notation:

$$
\mathbf{u}=\left[\begin{array}{l}
u_{r} \\
u_{\theta}
\end{array}\right]=-\frac{3}{4} \frac{a U}{r^{3}}\left[\begin{array}{l}
2 \cos \theta \\
-\sin \theta
\end{array}\right]
$$

using a stokeslet feeding current as an example. Here, $u_{\mathrm{r}}$ is the velocity in the radial direction and $u_{\mathrm{q}}$ is the velocity in the tangential direction

\begin{tabular}{|c|c|c|c|}
\hline Description & Equation & & Notes \\
\hline \multicolumn{4}{|l|}{ Low Reynolds number } \\
\hline Passively sinking particle & $\mathbf{u}=U\left[\begin{array}{c}-\cos \theta \\
\sin \theta\end{array}\right]+\frac{3}{4} \frac{a U}{r}\left[\begin{array}{c}2 \cos \theta \\
-\sin \theta\end{array}\right]-\frac{1}{4} \frac{a^{3} U}{r^{3}}\left[\begin{array}{c}2 \cos \theta^{-} \\
\sin \theta\end{array}\right.$ & (a1) & $\begin{array}{l}\text { For a spherical particle with radius a } \\
\text { and settling velocity } U \text {. Particle travels } \\
\text { in the } \theta=0 \text { direction (Leal 1992, Berg } \\
\text { 1993) }\end{array}$ \\
\hline Self-propelled body & $\mathbf{u}=U\left[\begin{array}{c}-\cos \theta \\
\sin \theta\end{array}\right]-\frac{3}{2} \frac{a^{2} U}{r^{2}}\left[\begin{array}{c}1-3 \cos ^{2} \theta \\
0\end{array}\right]$ & (a2) & $\begin{array}{l}\text { For a spherical body with radius } a \text { and } \\
\text { translation velocity } U \text {, moving in the } \theta= \\
0 \text { direction (Leal 1992) }\end{array}$ \\
\hline $\begin{array}{l}\text { Feeding current for } \\
\text { gravitationally tethered } \\
\text { \#ism: Eq. (a3), leading } \\
\text { stokeslet term; } \\
\text { Eq. (a4), spherical pump }\end{array}$ & $\begin{array}{l}\mathbf{u}=\frac{3}{4} \frac{a U}{r}\left[\begin{array}{l}2 \cos \theta \\
-\sin \theta\end{array}\right] \\
\mathbf{u}=-\frac{3}{4} \frac{a U}{r}\left[\begin{array}{l}2 \cos \theta \\
-\sin \theta\end{array}\right]-\frac{1}{4} \frac{a^{3} U}{r^{3}}\left[\begin{array}{c}2 \cos \theta \\
\sin \theta\end{array}\right]\end{array}$ & (a3) & $\begin{array}{l}\text { For a spherical equivalent drag where } \\
a U=g \Delta M /(6 \pi \mu) \text { where } \Delta M \text { is the } \\
\text { effective mass of the organism. (e.g. } \\
\text { Jonsson \& Tiselius 1990) } \\
\text { Spherical pump simulates uniform } \\
\text { velocity } U \text {, directed aft }\left(\theta=180^{\circ}\right) \text {, over a } \\
\text { spherical volume of radius } a . \text { (e.g. Kiør- } \\
\text { boe \& Visser 1999) }\end{array}$ \\
\hline $\begin{array}{l}\text { Flow disturbance } \\
\text { generated by a solid } \\
\text { particle, in a general linear } \\
\text { flow field }\end{array}$ & $\mathbf{u}^{\prime}=-\frac{a^{5}}{r^{5}} E \cdot \mathbf{r}-\frac{5}{2}\left(\frac{a^{3}}{r^{5}} \frac{a^{5}}{r^{7}}\right) \mathbf{r}(\mathbf{r} \cdot E \cdot \mathbf{r})-\frac{1}{2} \frac{a^{3}}{r^{3}} \omega \times \mathbf{r}$ & (a5) & $\begin{array}{l}E \text { is the rate of strain matrix, elements } \\
\text { for which in spherical polar coordinates } \\
\text { are listed in Batchelor (1967, p. 601), and } \\
\omega \text { is the vorticity of the undisturbed fluid } \\
\text { flow (Leal 1992) }\end{array}$ \\
\hline $\begin{array}{l}\text { Simple deformation flow; } \\
\text { e.g. solid particle in a }\end{array}$ & $u_{r}{ }^{\prime}=e a^{3}\left(3 \cos ^{2} \theta-1\right)\left(3 a^{2}-5 r^{2}\right) /\left(4 r^{4}\right)$ & (a6) & $\begin{array}{l}\text { Special case of Eq. (a5) for a simple } \\
\text { deformation field with: }\end{array}$ \\
\hline feeding current & $u_{\theta}{ }^{\prime}=e 3 a^{3} \sin \theta \cos \theta /\left(2 r^{4}\right)$ & $(\mathrm{a} 7)$ & $E=\frac{e}{2}\left(\begin{array}{ccc}2 & 0 & 0 \\
0 & -1 & 0 \\
0 & 0 & -1\end{array}\right) ; e=\frac{\partial u_{r}}{\partial r}$ \\
\hline Rotating flow or particle & $u_{\phi}{ }^{\prime}=-\Omega a^{3} \sin \theta / r^{2}$ & (a8) & $\begin{array}{l}\Omega \text { is the rotation of the particle with } \\
\text { respect to the undisturbed fluid flow. } \\
\text { Axis of rotation is in the } q=0 \text { direction. } \\
\text { Other components of the flow are zero. } \\
\text { (Leal 1992) }\end{array}$ \\
\hline \multicolumn{4}{|l|}{ Acoustic fields } \\
\hline $\begin{array}{l}\text { Acoustic dipole } \\
\text { Eq. (a9): near field, } \\
k r \ll 1 \\
\text { Eq. (a10): intermediate } \\
\text { field, } k r \approx 1 \\
\text { Eq. (a11): far field } \\
\text { (sound wave), } k r \gg 1\end{array}$ & $\begin{array}{l}\mathbf{u}=\frac{a^{3}}{2 r^{3}} U \cos \sigma t\left[\begin{array}{c}2 \cos \theta \\
\sin \theta\end{array}\right] \\
\mathbf{u}=\frac{k a^{3}}{2 r^{3}} U \sin (\sigma t-k r)\left[\begin{array}{c}2 \cos \theta \\
\sin \theta\end{array}\right] \\
\mathbf{u}=\frac{k^{2} a^{3}}{2 r} U \cos (\sigma t-k r)\left[\begin{array}{c}\cos \theta \\
0\end{array}\right]\end{array}$ & $\begin{array}{r}\text { (a9) } \\
\text { (a10) }\end{array}$ & $\begin{array}{l}\text { Generated by a vibrating body of radius } \\
\text { a, maximum velocity } U \text { and angular } \\
\text { frequency } \sigma \text {. The speed of sound, } C \text {, lies } \\
\text { in the range } 1450 \text { to } 1550 \mathrm{~m} \mathrm{~s}^{-1} \text { for } \\
\text { marine waters. Wave number, } k \text {, is } \\
k=\sigma / C \text { (Lighthill } 1978 \text {, Kalmijn 1988) }\end{array}$ \\
\hline \multicolumn{4}{|l|}{ High Reynolds number } \\
\hline Potential flow & $\mathbf{u}=U\left[\begin{array}{c}-\cos \theta \\
\sin \theta\end{array}\right]+\frac{1}{2} \frac{a^{3} U}{r^{3}}\left[\begin{array}{c}2 \cos \theta \\
\sin \theta\end{array}\right]$ & $(\mathrm{a} 12)$ & $\begin{array}{l}\text { Around a sphere of radius } a \text {, and veloc- } \\
\text { ity } U \text { in the } \theta=180^{\circ} \text { direction. Valid for } \\
r>O\left(a R e^{-1 / 2}\right) \text { (Batchelor 1967) }\end{array}$ \\
\hline
\end{tabular}


Table 3 (continued)

\begin{tabular}{|c|c|c|c|}
\hline Description & Equation & & Notes \\
\hline $\begin{array}{l}\text { Viscous wake behind a } \\
\text { translating body }\end{array}$ & $\mathbf{u}=\frac{Q U}{4 \pi v} \frac{1}{r} \exp \left\{-\frac{U}{4 v} r \frac{\sin ^{2} \theta}{\cos \theta}\right\}\left[\frac{2+\tan ^{2} \theta}{-\tan \theta}\right]$ & (a13) & $\begin{array}{l}\text { Body travelling at velocity } U \text { in the } \theta= \\
180^{\circ} \text { direction. The parameter } Q \text { is } \\
\text { given by } Q=U A_{X} C_{D} / 2 \text { (Batchelor 1967) }\end{array}$ \\
\hline $\begin{array}{l}\text { Equation of motion } \\
\text { for a rigid particle in a } \\
\text { general irrotational fluid flow }\end{array}$ & $\begin{array}{l}\left(\gamma+C_{M}\right) \frac{\mathrm{d} \mathbf{u}}{\mathrm{d} t}=(\gamma+1) \frac{\mathrm{d} \mathbf{v}}{\mathrm{d} t}+(\gamma-1) \mathbf{g}-\frac{\mathbf{F}_{\mathrm{D}}}{\rho_{f} V_{P}} \\
\mathbf{u}=\text { relative velocity of the particle } \\
\mathbf{v}=\text { fluid motion } \\
\mathbf{g}=\text { acceleration due to gravity }\end{array}$ & (a14) & $\begin{array}{l}C_{\mathrm{M}} \text { is the added mass coefficient and } \\
\text { equals } 1 / 2 \text { for a sphere; } \gamma=\rho_{P} / \rho_{f} \text { : } \\
\text { the ratio of the particle to fluid density } \\
\text { and } V_{p} \text { is the particle volume } \\
\text { (Maxey \& Riley 1983, Fung 1998) }\end{array}$ \\
\hline $\begin{array}{l}\text { Drag on a smooth moving } \\
\text { body } \\
\text { Eq. (a15): both High and } \\
\text { Low } R e \\
\text { Eq (a17): sphere of radius a } \\
\text { at low } R e\end{array}$ & $\begin{array}{l}\mathbf{F}_{\mathrm{D}}=U 2 \rho A_{x} \mathrm{C}_{\mathrm{D}} / 2 \hat{x} \\
\mathrm{C}_{\mathrm{D}}(R e)=\frac{24}{R e}\left(1+0.0315 R e^{(0.82-0.05 U)}\right) \\
\mathbf{F}_{\mathrm{D}}=6 \pi a \mu U \hat{x}\end{array}$ & $\begin{array}{l}\text { (a15) } \\
\text { (a16) }\end{array}$ & $\begin{array}{l}A_{X} \text { is the cross-sectional area of the } \\
\text { body perpendicular to the direction of } \\
\text { motion, } \hat{\mathbf{x}} \text {. The drag coefficient, } C_{D} \text { is for } \\
\text { a spherical body, spanning low to high } \\
\text { Reynolds numbers: } R e=2 U a / \mu \\
\text { (Eq. a15: Batchelor } 1967 \text {; Eq. a16: Clift } \\
\text { et al. 1978) }\end{array}$ \\
\hline
\end{tabular}

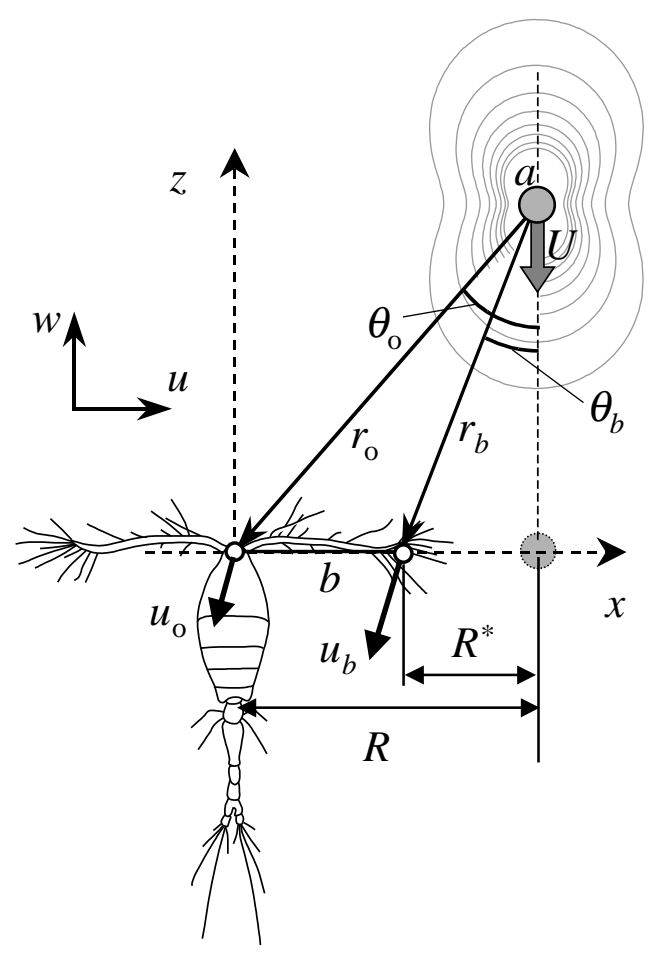

Fig. 4. Detection of a settling particle (radius a, settling velocity $U$ ) by a raptorial copepod (antenna length $b$ ). As a simplification, the centre of mass of the copepod is at $\left(r_{0}, \theta_{0}\right)$ measured from the centre of mass of the settling particle. The copepod tends moves as a whole at a velocity $u_{0} . u_{b}$ is the velocity at the tip of the antenna $\left(r_{b}, \theta_{b}\right)$, and detection occurs when $\left|u_{0}-u_{b}\right|$, the absolute velocity difference at $b$ exceeds the sensitivity threshold for the copepod, $s$. The direction of the Cartesian axes $(x, Z)$ and velocity components $(u, w)$ are indicated. A case is represented whereby the sinking particle is detected on the $x$-axis: $R$ is the detection distance between the centres of mass of the copepod and settling particle, and $R^{*}$ is the detection distance between the centre of mass of the settling particle and a single detector given by:

$$
\mathbf{u}_{\mathrm{o}}=\frac{3}{4} \frac{a U}{r_{\mathrm{o}}}\left[\begin{array}{c}
2 \cos \theta_{\mathrm{o}} \\
-\sin \theta_{\mathrm{o}}
\end{array}\right]-\frac{1}{4} \frac{a^{3} U}{r^{3}}\left[\begin{array}{c}
2 \cos \theta_{\mathrm{o}} \\
\sin \theta_{\mathrm{o}}
\end{array}\right]
$$

where $\left(r_{0}, \theta_{0}\right)$ is the position of the copepod relative to the sinking particle (Fig. 4). A seta positioned somewhat closer to the settling body at $\left(r_{b}, \theta_{b}\right)$ will be at a location where the ambient fluid velocity, $u_{b}$, is different. The seta will therefore experience an absolute velocity difference, $\delta \mathbf{u}=\mathbf{u}_{b}-\mathbf{u}_{0}$. In the simplest case, where the particle is closest to the copepod (i.e. $\theta_{0}=$

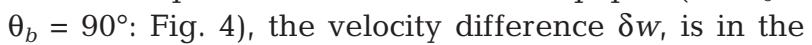
vertical direction and given by:

$$
\delta w=\frac{3 U}{4} \frac{a b}{r(r-b)}+\frac{3 U}{4} \frac{a^{3} b}{r^{2}(r-b)^{2}}+\frac{U}{4} \frac{a^{3} b^{3}}{r^{3}(r-b)^{3}}
$$

Assuming that detection occurs when $\delta w$ exceeds a given sensitivity, $S$, the distance $R^{*}$ at which the copepod detects can be deduced from Eq. (10). However, it is more convenient to consider 2 limits; $R^{*} \gg b$ and $R^{*} \ll b$, i.e. when the detection distance is much greater than, and much less than the detector length scale. These are given by:

$$
\begin{array}{ll}
R^{*} \ll b & R^{*}=\frac{1}{4} \frac{U}{S} a b\left(3+\frac{a^{2}}{R^{* 2}}\right) \\
R^{*} \gg b & R^{* 2}=\frac{3}{4} \frac{U}{S} a b\left(1+\frac{a^{2}}{R^{* 2}}\right)
\end{array}
$$

Firstly, if $R^{*}>$ a $\left(R^{*} \leq a\right.$ means physical contact of the particle with the sensor), to a first approximation the solution becomes $R^{*} \approx 3 / 4 U a S^{-1}$ for $R^{*} \ll b$; in this case the solid-body motion of the copepod is negligible and the velocity at the setae depends only on the setae's distance from the centre of the settling body. Secondly, 
if $R^{*} \gg b$, the solution becomes $R^{*} \approx\left(3 / 4 U a b s^{-1}\right)^{1 / 2}$; in this case, the motion of the copepod induced by the falling body is important, and the detection distance becomes dependent on the gradient of the flow disturbance (the rate of strain) and the length scale of the detector. These 2 limits are essentially those for the prey perceiving the predator and vice versa (Kiørboe \& Visser 1999). The pertinent parameter depends not only on the relative size of the prey and predator $(a / b)$, but also on the relevant velocity scales $(U / S)$. This can be demonstrated by rewriting Eq. (10) for $R^{*}=r-b>a$ as:

$$
R^{*}=\frac{b}{2}\left(\left(1+3 \frac{a}{b} \frac{U}{s}\right)^{1 / 2}-1\right)
$$

The detection and ingestion of settling particles such as marine snow and faecal pellets by copepods and other zooplankton is thought to be an important factor controlling the net vertical flux of carbon in the open ocean (e.g. Jackson 1993, González \& Smetacek 1994). Both chemoreception (González \& Smetacek 1994) and mechanoreception (Kiørboe \& Visser 1999) are thought to be agents promoting this type of encounter rate. Regarding mechanoreception, we note that the settling velocity $U$ is also dependent on particle size $a$. For example, relatively compact particles such as faecal pellets settle at a rate determined by Stokes law, i.e. $U=2 g a^{2} \Delta \rho /(9 \mu)$, where $g=980 \mathrm{~cm} \mathrm{~s}^{-2}, \mu=10^{-2} \mathrm{~g} \mathrm{~cm}^{-1} \mathrm{~s}^{-1}$ and $\Delta \rho=$ the density difference between the fluid and the particle. This implies a particle size limit below which there is no detection, i.e. when $R^{*} \leq a$, the particle cannot be detected, leading to the size limit $a_{0}$ :

$$
a_{0}=\left(\frac{6 \mu s}{g \Delta \rho}\right)^{1 / 2}
$$

For Oithona similis $\left(b=0.75 \mathrm{~mm}, s=40 \mu \mathrm{m} \mathrm{s}^{-1}\right)$ feeding on faecal pellets of $1.15 \mathrm{~g} \mathrm{~cm}^{-3}$ density, this gives $a_{0}=13 \mu \mathrm{m}$, which is about half the size of an Acartia tonsa faecal pellet $(a=25 \mu \mathrm{m})$. Fig. 5 a shows $R^{*}$ as a function of faecal pellet size. Here we can see 2 distinct functional forms: $R^{*} \propto a^{3 / 2}$ and $R^{*} \propto a^{3}$, for when the motion of the copepod is and is not important, respectively.

In Fig. $5 \mathrm{~b}$ we see how this perceptive distance influences the clearance rate $\beta$. This is given by the product of velocity and the cross-sectional area enclosed by the distance $R^{*}$ from the antenna. Specifically,

$$
\beta=U(a)\left(4 b R^{*}(a)+\pi R^{*}(a)^{2}\right)
$$

Fig. 5 shows that $\beta \propto a^{5}$, or at least very nearly so. This is because the transition from $R^{*} \propto a^{3}$ to $R^{*} \propto a^{3 / 2}$ in Fig. 5a corresponds very nearly to the transition from an essentially rectangular $\left(\propto R^{*}\right)$ to a circular $\left(\propto R^{* 2}\right)$ cross-sectional area. The strong dependence of clearance rate on particle size suggests a strong selectivity for large particles. For other, less compact marine
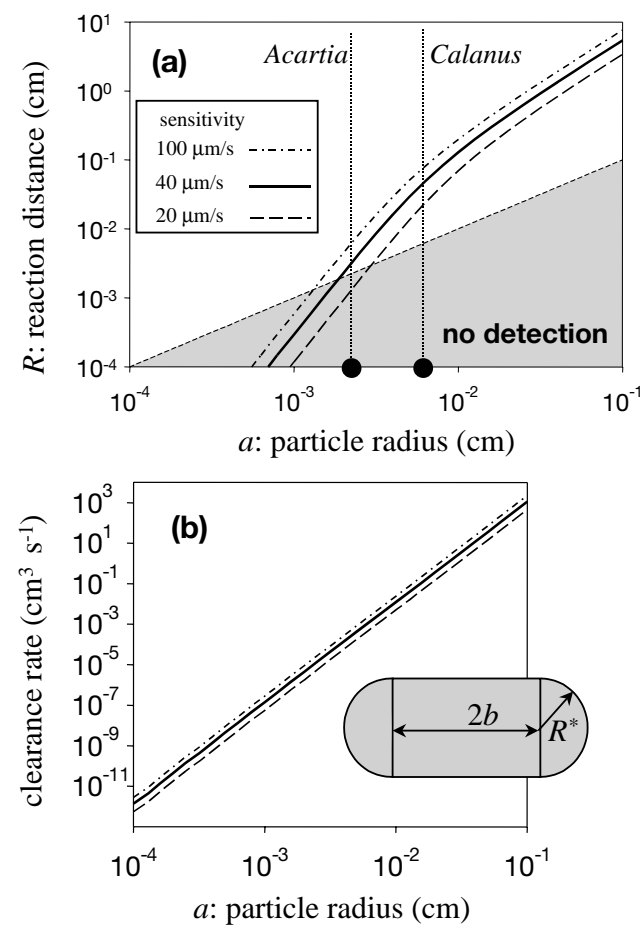

Fig. 5. Detection distance of the hydromechanical signal generated by a settling particle (radius a) following Stokes law. Parameters are representative for faecal pellets (density $1.15 \mathrm{~g} \mathrm{~cm}^{-3}$ ) being detected by Oithona similis (antenna length, $b=0.75 \mathrm{~cm}$ ). (a) Three detection curves plotted for sensitivity threshold $s=20,40$ and $100 \mu \mathrm{m} \mathrm{s}^{-1}$; region of 'no detection' is where physical contact is made before the threshold sensitivity is exceeded; the 2 vertical lines indicate representative sizes for Acartia tonsa and Calanus sp. faecal pellets respectively. (b) Estimated clearance rate $\beta\left(\mathrm{cm}^{3} \mathrm{~s}^{-1}\right)$ calculated from the cross-sectional area of the detection zone and the particle settling velocity; inset: dependence of crosssectional area on antenna length and detection distance

particles and aggregates, Eq. (12) may overestimate their vertical velocity. Jackson (1990) suggested that phytoplankton cells' settling velocity scales as $U \propto a^{1.17}$ due to a decrease in density with increasing size. Even with this much weaker size dependence on settling, the clearance rate is still considerably biased towards large particles: $\beta \propto a^{3.34}$.

\section{Self-propelled body}

A large class of zooplankton are motile (copepods, nauplii, ciliates, etc.), and evidence suggests that mechanoreception plays a major role in remote detection of these as prey (Landry 1980, Williamson \& Vanderploeg 1988, Jonsson \& Tiselius 1990, Svensen \& Kiørboe 2000).

The net force exerted on the fluid by a neutrallybuoyant, self-propelled body moving at uniform 


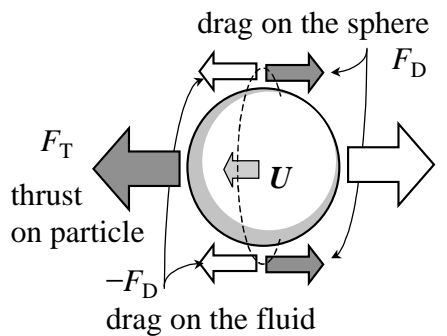

drag on the fluid

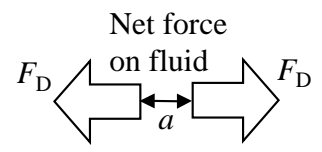

Fig. 6. Forces exerted on a fluid by a self-propelled spherical

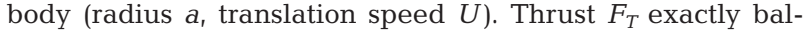
ances drag $F_{D}$ for steady translation. Both these forces have equal and opposite influence on the fluid; thus the flow field is approximated by a stresslet to first order

velocity, $U$, is zero. That is, the thrust driving the body forward is exactly balanced by the viscous drag on the body. Both thrust and drag act on the fluid as well so that the forces exerted on the fluid are also equal and directed in opposite directions. This is quite different from an organism or particle that moves under the influence of a body force (e.g. gravity) where only drag acts on the fluid. Thus, the far-field flow for a self-propelled body tends to that of a force dipole. A simple case is a self-propelled sphere of radius $a$, where the thrust is applied to the surface of the sphere and acts through its centre of mass (Fig. 6). While the drag acts over the surface of the sphere, we can, to a first-order, consider it as an effective point force diametrically opposed to thrust. The strength of the dipole forces are thus $\left|\mathbf{F}_{\mathrm{D}}\right|=6 \pi \mu a U_{\text {; }}$ they are equal and opposite, and are separated by distance 2a. Substituting into Eq. (4) leads to:

$$
\begin{aligned}
& u_{r}=\frac{3}{2} \frac{U a^{2}}{r^{2}}\left(1-3 \cos ^{2} \theta\right) \\
& u_{\theta}=0
\end{aligned}
$$

and taking into account the translation of the body with respect to the far-field fluid leads to Eq. (a2) in Table 3.

In general, the velocity difference between a sensor at $\left(r_{b}, \theta_{b}\right)$ and the centre of mass of a copepod at $\left(r_{0}, \theta_{0}\right)$ (Fig. 7) is given by:

$$
\begin{aligned}
& \delta \mathbf{u}=\mathbf{u}_{b}-\mathbf{u}_{\mathrm{o}}= \\
& \frac{3}{2} \frac{U a^{2}}{r_{b}{ }^{2}}\left(1-3 \cos ^{2} \theta_{b}\right) \hat{r}_{b}-\frac{3}{2} \frac{U a^{2}}{r_{\mathrm{o}}{ }^{2}}\left(1-3 \cos ^{2} \theta_{\mathrm{o}}\right) \hat{r}_{\mathrm{o}}
\end{aligned}
$$

The vectors $\hat{r}_{\mathrm{o}}$ and $\hat{r}_{b}$ are the unit radial vector evaluated at $o$ and $b$ respectively. Assuming that detection occurs when $|\delta \mathbf{u}| \geq S$, the detection distance $R^{*}$, can be deduced from Eq. (16). However, the general case is algebraically cumbersome. Instead, consider first the case in which detection occurs close to the detector. Specifically, $r_{b}=R^{*} \ll b$. This necessarily means that $r_{\mathrm{o}}=R \gg R^{*}$. In this case, the velocity at $b$ dominates in Eq. (16), and the detection distance is given by:

$$
R^{*}<b: \quad R^{*}=a\left(\frac{3}{2} \frac{U}{S}\left|3 \cos ^{2} \theta-1\right|\right)^{1 / 2}
$$

On the other hand, when the detection distance is large compared to the antenna size $b$, we have $\theta_{\mathrm{o}} \cong \theta_{b}(=\theta)$, and $r_{\mathrm{o}}^{2} \cong r_{b}^{2}+2 r_{b} b \cos (\theta-\xi)$. The detection distance $R^{*}$ is given by:

$$
R^{*}>b: \quad R^{*} \cong\left(3 a^{2} b \frac{U}{S}\left|3 \cos ^{2} \theta-1\right| \cos (\theta-\xi)\right)^{1 / 3}
$$

where $\xi$ is the angle between the approach direction and the alignment of antenna (Fig. 7). The conditions to which these scaling rules apply can be specified by noting the magnitude of the estimates. Specifically, Eq. (17) applies when $a U^{2} /\left(b s^{2}\right)<1$, whereas Eq. (18) applies when $a U^{2} /\left(b s^{2}\right)>1$.

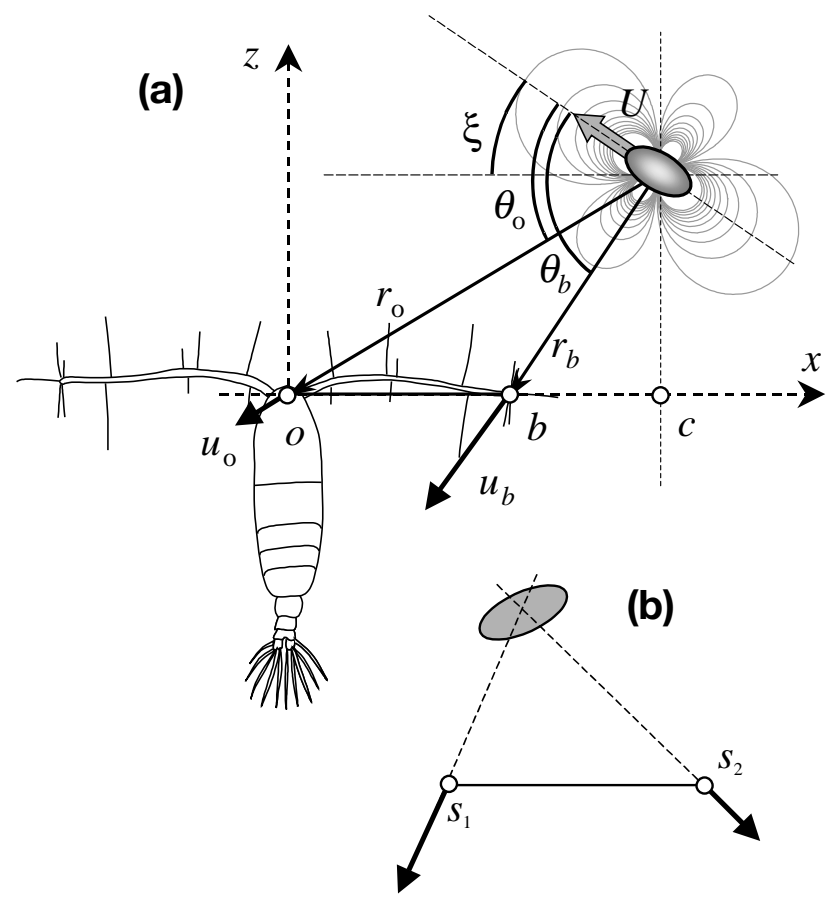

Fig. 7. (a) Detection of a self-propelled body (effective radius $a$, speed $U$ ) by a copepod of antenna length $b$; for simplicity, the centre of mass of the copepod is assumed to be at $\left(r_{0}, \theta_{0}\right)$ and a detector at the end of the antenna at $\left(r_{b}, \theta_{b}\right)$; these are measured relative to the centre of mass and direction of motion of the swimming organism; $\xi$ : the angle between direction of motion and the antenna; a special case is considered whereby the self-propelled organism is on the $x$-axis.

(b) Location of a self-propelled body by triangulating the direction of velocity disturbance at 2 setae, $s_{1}$ and $s_{2}$ 


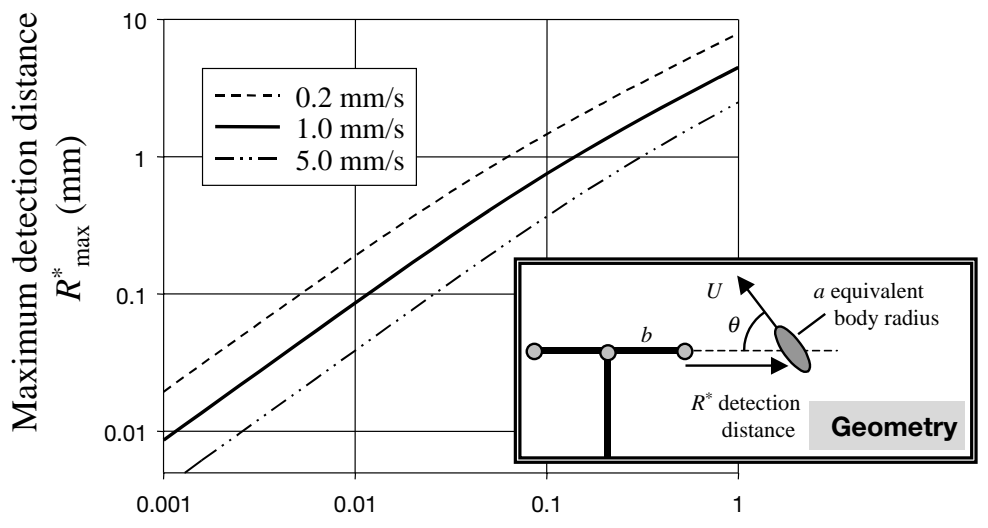

Size of self-propelled organism, $a(\mathrm{~mm})$

Fig. 8. Maximum detection distance $R^{*}{ }_{\max }$ as a function of the equivalent spherical radius of a self-propelled organism. Curves are plotted for 3 different translational velocities, $U=0.2,1$ and $5 \mathrm{~mm} \mathrm{~s}^{-1}$. The sensitivity $s=40 \mu \mathrm{m} \mathrm{s}^{-1}$ and antenna length $b=1 \mathrm{~mm}$. The curves are for the particular detector-translation alignment shown in inset. Actual detection distance $R^{*}$ depends on the angle of approach, $\theta: R^{*}=1 / 2 R^{*}{ }_{\max }\left|3 \cos ^{2} \theta-1\right|$

\section{Directional information}

For both settling particles and swimming organisms, the assumed criterion for detection is simply that the absolute velocity difference at the detector, $\left|\mathbf{u}_{0}-\mathbf{u}_{b}\right|$, exceeds a detection threshold, $s$. While this approach certainly indicates the feasibility of detection, it says nothing about higher information content that can be used in locating the source of the hydromechanical signal. Copepod setae appear to be attuned to measure velocity disturbances in different directions (Yen et al. 1992); for instance, some setae have hinges that allow them to bend only in particular directions. Given this, then the possibility of location abilities exists. The simplest case is that of locating a small swimming organism. Because the velocity disturbance is always radial, if a copepod can measure the direction of the velocity dis-

The dependence of detection distance on the size and speed of a self-propelled organism is plotted in Fig. 8 . The velocity difference between the centre of the copepod, and the end of its antenna is calculated using Eq. (16) for a self-propelled organism crossing the equatorial plane of the detector (at $c$ in Fig. $7 ; \theta=$ $\left.\theta_{0}=\theta_{b}=\xi, r_{0}=r_{b}+b\right)$. The velocity difference is:

$$
\frac{\left(R^{*}+b\right)^{2} R^{* 2}}{2 R^{*}+b}=\frac{3}{2} \frac{U}{S} a^{2} b\left|1-3 \cos ^{2} \theta\right|
$$

where $R^{*}$ is the distance from the self-propelled organism to the antennular tip.

Jonsson \& Tiselius (1990) reported observations of the reaction distance of the copepod Acartia tonsa feeding on motile ciliates. In particular, when feeding on Loboea strobila (length $=130 \mu \mathrm{m}$, width $=65 \mu \mathrm{m}$ ), a ciliate with swimming speed, $U$, from 0.5 to $1 \mathrm{~mm} \mathrm{~s}^{-1}$, a detection distance of about $0.5 \mathrm{~mm}$ was observed. In comparison, when feeding on Strombidium reticulatum (length $=43 \mu \mathrm{m}$, width $=30 \mu \mathrm{m}, U=0.5$ to $1 \mathrm{~mm}$ $\mathrm{s}^{-1}$ ), the observed detection distance was considerably less, with nearly all attacks occurring at less than $0.25 \mathrm{~mm}$. Substituting these values into Eq. (17) and assuming $s=40 \mu \mathrm{m} \mathrm{s}^{-1}$ gives reaction distances of 0.40 to $0.56 \mathrm{~mm}$ and 0.13 to $0.19 \mathrm{~mm}$ for Loboea strobila and Strombidium reticulatum respectively. More recently, Svensen \& Kiørboe (2000) reported observed reaction distances $R=140 \pm 70 \mu \mathrm{m}$ for Oithona similis feeding on Gymnodinium dominans $(a=19 \mu \mathrm{m}$, $U=0.58 \mathrm{~mm} \mathrm{~s}^{-1}$ ). Again, substituting into Eq. (17) we get $R=125 \mu \mathrm{m}$. In all instances, estimated and observed detection distances correspond relatively well. turbance at the same time at 2 locations along its antenna, simple triangulation would point directly back to the swimming organism (Fig. 7b).

\section{Feeding current}

Feeding currents are a behavioural adaptation of many zooplankton species, including copepods and ciliates. In copepods, these feeding currents are usually generated by movements of the second antennae, first maxillae and the maxillipeds or combinations thereof (e.g. Koehl \& Strickler 1981, Paffenhöfer et al. 1982, Strickler 1982). The general shape of the feeding current is a funnel-like, inwardly-directed flow in front of the animal (Strickler 1985, Tiselius \& Jonsson 1990).

The simplest physical model of a feeding current is that of a gravitationally tethered copepod, the thrust of whose feeding current exactly balances its negative buoyancy (Strickler 1982, Emlet \& Strathman 1985). As with a sinking particle, a feeding current exerts a net force on the fluid (Fig. 9); this leads immediately to Eq. (a3) in Table 3, which is the stokeslet model of a copepod feeding current introduced by Tiselius \& Jonsson (1990). The spherical pump model (Kiørboe \& Visser 1999) is a slight variation of this (Eq. a4: Table 3) whereby the thrust is distributed over a finite spherical volume of space.

The force balance as illustrated in Fig. 9 is a firstorder approximation. Higher-order effects can be important close to the source. In particular, the flow generated by thrust also exerts a downward drag on the body, $\mathbf{F}_{\mathrm{D}}$ (Emlet \& Strathman 1985). The thrust $\mathbf{F}_{\mathrm{T}}$ 


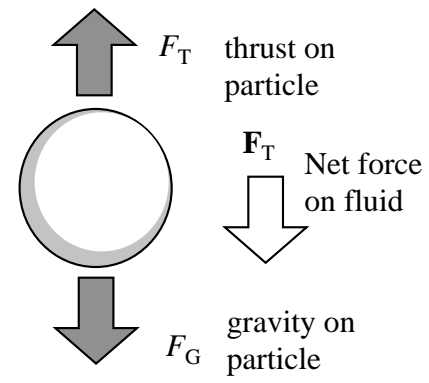

Fig. 9. Force exerted on fluid by a gravitationally tethered organism generating a feeding current. Thrust $F_{\mathrm{T}}$ exerted on the particle exactly balances the negative buoyancy of the $\operatorname{organism} F_{\mathrm{G}}$. Gravity, however, is a body force acting only on the organism, so that the net force exerted on the fluid is $F_{\mathrm{T}}$ downwards. This suggests that, to first-order, the flow associated with the feeding current is a stokeslet of strength $F_{\mathrm{T}}$

must therefore be in excess of gravity $\mathbf{F}_{\mathrm{G}}$ by an amount $\mathbf{F}_{\mathrm{D}}$. Thus the feeding current close to the source (within about a body length) is more properly modelled as a stokeslet of strength $\mathbf{F}_{\mathrm{G}}$, and a stresslet of strength $\mathbf{F}_{\mathrm{D}}$, distributed over half a body length. The combination of a stokeslet plus a stresslet generates a feeding current which has a fore-aft asymmetry.

The feeding current structure suggested by these different models (pure stokeslet, spherical pump, stokeslet + stresslet) each approach an $r^{-1}$ fall-off rate for $r \gg a$ (Fig. 10). However, close to the copepod's

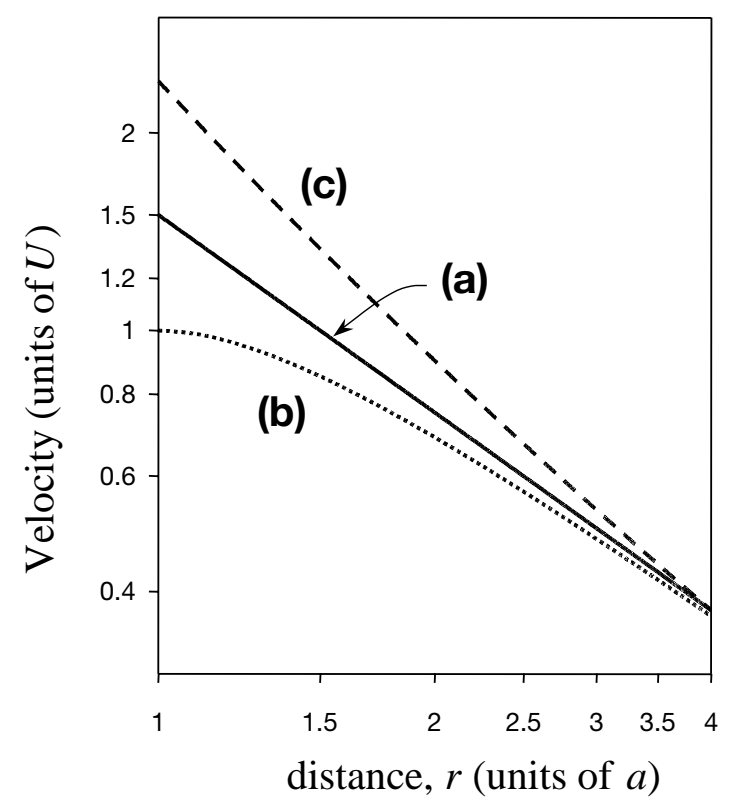

Fig. 10. Three models for feeding current structure (velocity scale $U$ and effective radius a). The pure stokeslet (a) has a $r^{-1}$ dependence, the spherical pump (b) approaches a flatter spatial dependence close to source, and the stokeslet + stresslet model (c) has a steeper dependence close to the source body, the spherical pump model has a flatter spatial dependence, whereas the effect of drag is to steepen the slope towards an $r^{-2}$ dependence. While to a large degree physiology is an important determining factor, one can expect the copepod to have some behavioural control over which of these feeding current structures it will generate. For instance, a copepod can control the rate at which it sweeps water downwards, as well as the reach and/or cross-sectional area through which it sweeps in such a way as to maintain the same net downward thrust. A copepod slowly pumping water across a relatively large cross-sectional area may present a 'spherical pump'-like profile. In contrast, a copepod rapidly pumping water through a small crosssection can generate considerable drag, presenting a 'stokeslet + stresslet' velocity profile.

There are several analyses of feeding current structure in the literature. Yen et al. (1991) analysed the feeding current of free-swimming Euchaeta rimana and found the velocity to follow $u \propto r^{-\mathrm{n}}$ with $n=1.5$ and 1.04 along 2 different paths approaching the feeding appendages. Similar analyses (Tiselius \& Jonsson 1990, Kiørboe \& Saiz 1995) showed a spread of $n$ from 0.38 to 1.67 for a variety of copepod species, with the mean being $n \approx 1$. In addition to copepods, other planktonic organisms' feeding currents would be expected to exhibit similar spatial attenuation. For instance, Gallager (1993) reports the flow field of both sinking and hovering bivalve larvae. Reanalysing his data gives $n=1.01\left(\mathrm{SE}=0.154, \mathrm{R}^{2}=0.82\right)$ when a bivalve larva is inactive and sinks, and $n=0.87\left(\mathrm{SE}=0.086, \mathrm{R}^{2}=0.72\right)$ when a larva hovers.

Detection of a copepod's feeding current by prey items and their subsequent escape ability are important factors driving prey selectivity in the plankton. Many aspects of this process have been investigated (Haury et al. 1980, Fields \& Yen 1997b, Kiørboe et al. 1999). I will not pursue this issue further in this work, focussing instead on the inverse process, namely on how the feeding current can alert the predator of the presence of a prey item entrained into it.

\section{Passive particles in a feeding current (predator-generated flow)}

Copepods can apparently detect non-motile particles being swept towards them in their feeding current while they are still several particle diameters away. While chemical signals can certainly be a cue for this detection, hydromechanical signals are also likely to play a role (Buskey 1984, Vanderploeg et al. 1990, DeMott \& Watson 1991, Bundy et al. 1998). LegierVisser et al. (1986) presented a theoretical treatment of mechanoreception. However, their work was some- 
what flawed. Firstly it considered pressure gradients as the active signal while, as pointed out by Bundy et al. (1998), velocity appears to be the more relevant signal. More seriously, however, Legier-Visser et al. (1986) failed to take into account the motion of the particle in the feeding current, i.e. they assumed the particle to be stationary with respect to the copepod, and moving with respect to the fluid. This is physically unrealistic for an inert planktonic particle and seriously overestimates the character and strength of the signal.

A neutrally buoyant particle in a flow field will in general accelerate at the same rate as would the fluid at its centre of mass. Only if a particle is more (less) dense, will it accelerate slower (faster) than the ambient fluid, a point addressed by Kiørboe \& Visser (1999). However, a neutrally-buoyant, rigid body embedded in a deforming or rotating flow field, can generate a local flow disturbance simply by its physical presence, the reason being that the flow must conform to having no deformation and no relative rotation on the boundary of the rigid particle - a condition that leads to a perturbation of the flow.

Eq. (a5) in Table 3 describes the flow field disturbance, $\mathbf{u}^{\prime}$, generated by a rigid spherical body located within a general deforming flow field. This flow perturbation can contribute to the remote detection of an otherwise inert plankton cell in a copepod feeding current. Consider a copepod of effective radius a generating a stokeslet feeding current (Eq. a3 in Table 3) of strength $U$ (Fig. 11). The rate of strain imposed on the fluid directly in front of the copepod $(\theta=0)$ is given by:

$$
e(r) \approx \frac{\delta u_{r}}{\delta r}=\frac{3}{2} \frac{a U}{r^{2}}
$$

where $r$ is the distance from the copepod. The perturbation field for this flow field (dominant term in Eq. a6 for $\theta=0$ : Table 3) in the direction of the principal axis of deformation is given by:

$$
u_{r}^{\prime}\left(r^{\prime}\right) \approx \frac{5}{2} e \frac{b^{3}}{r^{\prime 2}}
$$

where $b$ is the radius of the plankton cell, $e$ is the rate of strain at its position, and $r^{\prime}$ is the radial distance measured from the centre of the plankton cell. The plankton cell is detected when the perturbation velocity $u_{r}^{\prime}$ exceeds the threshold detection velocity $s$ at a sensor (seta) located on the first antenna. The distance from the plankton cell to the sensor can be approximated by $r^{\prime}=r-a$. This is a simplifying assumption, although it should be noted that $a$ is a hydrodynamical construction and not an anatomical measure. The separation distance, $R$, at which detection occurs is given by:

$$
s \approx \frac{15}{4} \frac{a b^{3} U}{R^{2}(R-a)^{2}}
$$

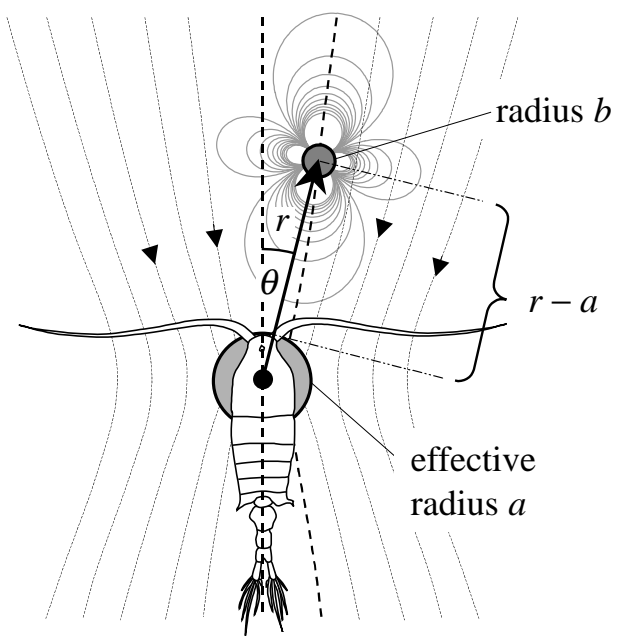

Fig. 11. Inert spherical particle, radius $b$, entrained into a copepod feeding current. The feeding current is modelled as a stokeslet flow with characteristic feeding current velocity, $U$, and effective solid body radius $a$. The feeding current flow field, $\mathbf{u}(r)$, is perturbed in the vicinity of the inert particle

which may be solved for $R$ as:

$$
R \approx \frac{a}{2}\left(1+\left(1+2\left(\frac{15 b^{3} U}{a^{3} s}\right)^{1 / 2}\right)^{1 / 2}\right)
$$

As an example, consider a copepod of equivalent radius $a=0.5 \mathrm{~mm}$ generating a feeding current of magnitude $U=10 \mathrm{~mm} \mathrm{~s}^{-1}$. The sensitivity $s$ is set at $0.02 \mathrm{~mm}$ $\mathrm{s}^{-1}$, consistent with measurements made by Yen et al. (1992). A plankton cell of radius $b=0.05 \mathrm{~mm}$ would then be detected at a distance of $R=0.89 \mathrm{~mm}\left(R^{*}=\right.$ 0.39 ).

Jonsson \& Tiselius (1990) report results for Acartia tonsa (length $800 \mu \mathrm{m}$ ) interacting with latex beads of $76 \mu \mathrm{m}$ diameter. While the nature of this interaction appeared to be a random collision rather than an active attack, this can be used as an example to test the feasibility of remote detection of inert particles in a feeding current. The maximum feeding current speed for A. tonsa is $8 \mathrm{~mm} \mathrm{~s}^{-1}$, dropping off to about $4 \mathrm{~mm} \mathrm{~s}^{-1}$ at $0.5 \mathrm{~mm}$ from the capture area (Jonsson \& Tiselius 1990). Taking $a=0.2 \mathrm{~mm}$ (ca half-width), $b=0.038 \mathrm{~mm}, U=8 \mathrm{~mm} \mathrm{~s}^{-1}$ and $\mathrm{s}=40 \mu \mathrm{m} \mathrm{s}^{-1}$ gives $R=0.42 \mathrm{~mm}\left(R^{*}=0.22 \mathrm{~mm}\right)$. It is tempting to compare this with the distance at which $A$. tonsa 'attacked' the latex beads in Jonsson \& Tiselius' (1990) study: about $0.3 \mathrm{~mm}$ from the copepod's head, although the exact process involved cannot be identified.

The limited number of direct observations means that definite conclusions cannot be drawn about this process. However, given known sensitivities, this study has demonstrated that the detection of inert particles in a feeding current is feasible. The conclusion is that, 


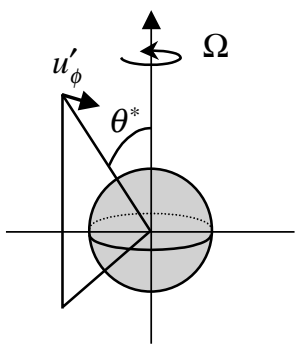

(a)

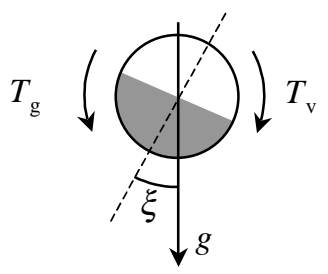

(b)

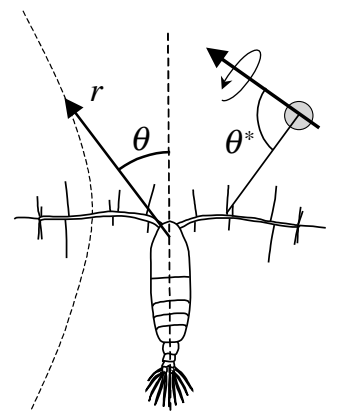

(c)
Fig. 12. Relative rotation. (a) Co-ordinates and velocity $\left(u_{\phi}^{\prime}\right)$ associated with a rotating sphere; (b) viscous $\left(T_{\mathrm{v}}\right)$ and gravitational $\left(T_{\mathrm{g}}\right)$ torque exerted on a sphere with an eccentric centre of mass; (c) co-ordinates for feeding current vorticity and detection of a self-rotating body

potentially, the feeding current is not just a means of sweeping food particles towards the feeding appendages, but can also serve as an integral part of the remote-sensing mechanism of a copepod.

\section{Relative rotation}

An inert spherical particle with uniform density rotates at the same rate as the ambient fluid. There are, however, 3 mechanisms that can lead to a relative rotation of an organism with respect to its ambient fluid. The first is by direct rotational swimming (e.g. rotifers). The second is through the interaction of a non-spherical shape in a deforming flow (Visser \& Jonsson 2000). Finally, relative rotation can result through a particle with an eccentric centre of mass interacting with ambient fluid rotation (e.g. Kessler 1986). In either case, the flow-field disturbance, $u$, generated by a rigid spherical particle of radius $b$ can be approximated by:

$$
u_{\phi}^{\prime}=-\Omega b^{3} \sin \theta^{*} r^{-2}
$$

(Eq. a8: Table 3), where $\Omega$ is the rate of rotation of the particle with respect to the fluid about the axis $\theta^{*}=0$.
The velocity field $u_{\phi}^{\prime}$ is directed perpendicular to the radial direction (Fig. 12a).

A feeding current generally has inherent vorticity, $\omega$. From the stokeslet model (Eq. a3, Table 3), one can estimate this to be:

$$
\omega=-3 a U \sin \theta r^{-2} / 2
$$

where $U$ is the feeding current speed and $a$ is the radial dimension of the pumping zone. This is effectively taken as half the copepod's body width.

A particle with an eccentric centre of mass entrained into a current with a horizontal component of vorticity will come under the influence of 2 torques: $T_{\mathrm{g}}$ due to gravity and $T_{\mathrm{v}}$ due to frictional drag arising from relative rotation with respect to the fluid (Fig. 12b). Assuming the particle to be spherical with radius $b$, these are given by:

$$
\begin{gathered}
T_{\mathrm{g}}=4 / 3 \pi \rho b^{3} g d \sin \xi \\
T_{\mathrm{v}}=8 \pi \mu b^{3} \Omega^{*}
\end{gathered}
$$

(Kessler 1986, Jonsson et al. 1991), where $\rho$ is the density of the particle, $d$ is the offset of the centre of mass from the geometric centre of the particle, $g$ is acceleration due to gravity, $\mu$ is the dynamic viscosity, and $\Omega^{*}$ is the relative rotation of the particle with respect to the fluid. Since the gravitational torque is dependent on $\xi$, the orientation of the particle with respect to the vertical, the particle can assume a steady orientation such that the gravitational and frictional torques balance. However, there is a limit to this balance. The maximum value the gravitational torque can achieve is $4 / 3 \pi \rho b^{3} g d$, and this happens when the relative rotation is $\Omega^{*}{ }_{\max }=$ $\rho d g /(6 \mu)$. That is, if the absolute fluid rotation is greater than $\Omega^{*}{ }_{\max }$, then the particle begins to tumble so that the relative rotations does not exceed is $\Omega^{*}{ }_{\max }$. Thus, given a sensitivity $s$, the detection distance for a biased particle in a feeding current has 2 forms depending on $\omega$, the vorticity of the feeding current, and the maximum particle rotation rate. Specifically,

$$
\begin{array}{ll}
\omega<2 \Omega^{*}{ }_{\text {max }}: & R^{*}\left(R^{*}+a\right)=\left(\frac{3 U a b^{3}}{4 s} \sin \theta\right)^{1 / 2} \\
\omega>2 \Omega^{*}{ }_{\text {max }}: & R^{*}=\left(\Omega^{*}{ }_{\max } b^{3} / s\right)^{1 / 2}
\end{array}
$$

Estimates of the separation distance, $d$, appear to be of the order of $1 \%$ of the radius of the organism (Fields \& Yen 1997a). This suggests a size-dependence on $\Omega^{*}{ }_{\text {max }}=\gamma b$, where $\gamma \approx 90 \mathrm{~cm}^{-1} \mathrm{~s}^{-1}$. For a typical copepod feeding current, (e.g. $a=0.2 \mathrm{~mm}, U=10 \mathrm{~mm} \mathrm{~s}^{-1}$ ), and its prey $(b<0.05 \mathrm{~mm})$, flow-field rotation nearly always exceeds $\Omega^{*}{ }_{\text {max }}$, so that Eq. (28) applies. That is $R^{*} \approx b^{2}$ $(\gamma / s)^{1 / 2}$. Thus, eccentric particles with radius $b<(s / \gamma)^{1 / 2}$ cannot be detected by their associated vorticity field. For $s=40 \mu \mathrm{m} \mathrm{s}^{-1}$, this gives $b_{\min }=66 \mu \mathrm{m}$. Particles larger than this can, in principal, be remotely detected, 
e.g. $b=100 \mu \mathrm{m}, s=40 \mu \mathrm{m} \mathrm{s}^{-1}$ gives $R^{*}=150 \mu \mathrm{m}$. Note that any flow disturbance generated by this mechanism will also have an associated deformation flow, as discussed previously ('Stokes flow solutions': 5th subsection). Given the analysis here, detection of the deformation field will in all likelihood occur at a greater distance than detection of the rotational field component.

In addition to passive rotation, some organisms actively rotate by applying a torque directly to the fluid. Ciliates and rotifers are examples, and both are potentially important food sources for copepods. For a raptorial copepod with sensitivity $s$, the distance at which it can detect a rotating organism of radius $b$, and angular velocity $\Omega$ is given by:

$$
R^{*}=\left(\Omega b^{3} \sin \theta^{*} / s\right)^{1 / 2}
$$

where $\theta^{*}$ is the angle between the axis of rotation and the vector from the centre of the rotating organism to the detector (Fig. 12c). Observations of rotation rates for swimming organisms are rare. Fenchel \& Jonsson (1988) reported an angular velocity of $\Omega \approx 0.5$ to 3.5 radian $\mathrm{s}^{-1}$ (i.e. 0.1 to 0.5 rotations $\mathrm{s}^{-1}$ ) for the ciliate Strombidium sulcatum $(b=15 \mu \mathrm{m})$. For this small, slowly rotating ciliate, detection is just possible at $R^{*}=17 \mu \mathrm{m}$ (for $s=40 \mu \mathrm{m} \mathrm{s}^{-1}$ ). Similar sizes and rotational rates for dinoflagellates (e.g. Prorocentrum mariae-labouriae) as reported by Kamykowski et al. (1992), lead to similar detection distances. However, larger, more rapidly rotating organisms can be detected at considerable distances. For example, a $50 \mu \mathrm{m}$ organism making 1 revolution $\mathrm{s}^{-1}\left(\Omega=2 \pi \mathrm{s}^{-1}\right)$ can be detected at a distance of $140 \mu \mathrm{m}$. The envelope of detection distances is plotted in Fig. 13.

\section{Passive particles approached by self-propelled predator}

To complete the examination of the detection of inert particles by hydromechanical signals, let us consider 1 final case, namely that of remote detection by a selfpropelled organism of a non-motile particle in its vicinity (Bundy et al. 1998). At a low Reynolds number, a self-propelled organism, such as a cruising copepod, generates a predominantly stresslet flow field which has inherent deformation and vorticity. Much as in the case of a feeding current ('Stokes flow solutions'; 5th subsection), an inert solid particle within a stresslet flow field generates a perturbation flow that can, in principle, be remotely detected. In order to explore the feasibility of this mechanism, I computed the rate of strain in front of a self-propelled organism $(\theta=0)$. From Eq. (15) and following Eq. (19), this is given by:

$$
e(r) \approx \frac{\partial u_{r}}{\partial r}=6 \frac{a^{2} U}{r^{3}}
$$

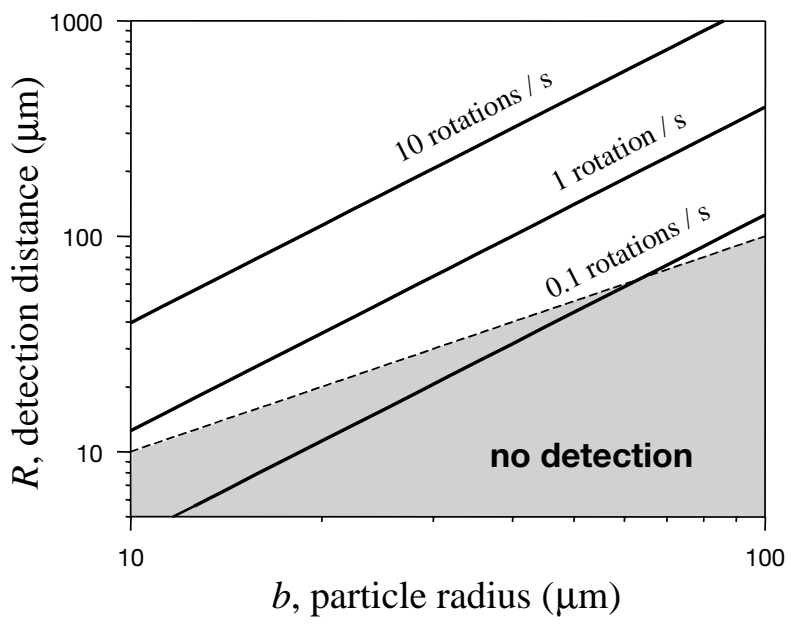

Fig. 13. Detection distance, $R$, of a rotating organism of radial dimension $b$. In this example, we assume that the detector is large compared to $b$, and detection is triggered when the velocity exceeds $40 \mu \mathrm{m} \mathrm{s}^{-1}$. The no detection zone is defined as when $R<b$. Three detection curves are plotted, for rotation rates of $0.1,1$ and 10 rotations $\mathrm{s}^{-1}$

Substituting into Eq. (20) and for a given sensitivity $S$, detection occurs at the distance $R$ given by the solution of:

$$
R^{3}(R-a)^{2}=15 a^{2} b^{3} \frac{U}{S}
$$

Observations by Bundy et al. (1998) of Diaptomus sicilis (length $1.2 \mathrm{~mm}$ ) attacking polystyrene beads (diameter $50 \mu \mathrm{m}$ ) appeared to follow this scenario. The copepod swam slowly towards the bead at a speed of 1 to $2 \mathrm{~mm} \mathrm{~s}^{-1}$ ( $R e=0.5$ to 1$)$. As the copepod approached, the bead began to move in advance of the copepod. At about $1 \mathrm{~mm}$ distance, the copepod attacked the bead. Setting $a=0.5 \mathrm{~mm}, b=0.025 \mathrm{~mm}, U=2 \mathrm{~mm} \mathrm{~s}^{-1}$ and $s=$ $0.02 \mathrm{~mm} \mathrm{~s}^{-1}$ gives $R=0.65 \mathrm{~mm}$. It appears feasible, therefore, that inert particles can be remotely detected by moving organisms due to a perturbation to their own flow field; i.e. as first suggested by Zaret (1980), being non-motile is no guarantee of being hydrodynamically inconspicuous.

\section{BEATING APPENDAGES AND ACOUSTIC SIGNALS}

Copepods and other zooplankton use beating appendages to propel themselves through the water or to generate feeding currents (Montgomery \& MacDonald 1987, Tiselius et al. 1997, van Duren 2000). The oscillatory signals associated with this behaviour have often been suggested as a means by which these organisms can be remotely detected (Kirk 1985). While copepods do not appear to be sensitive to pressure per se (Lenz \& Yen 1993, Bundy et al. 1998), they are sen- 


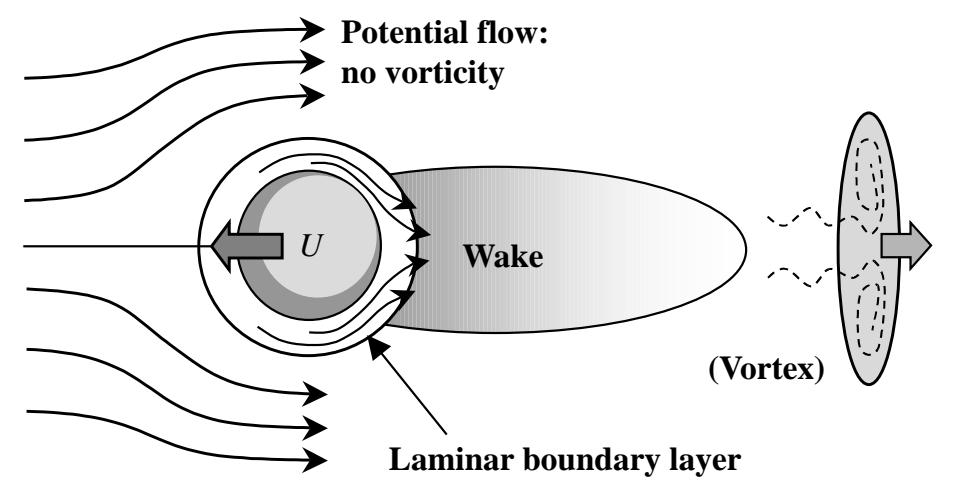

Fig. 14. The 4 hydrodynamic components associated with the translation of a body at high Reynolds number Re for a spherical body of radius a moving with velocity $U$. Laminar boundary-layer thickness is of the

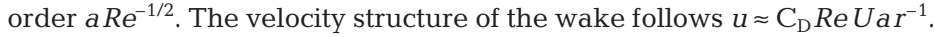
When the momentum imparted to the wake is sufficient, a vortex can form moving down-stream of the moving body. Up-stream, in an otherwise undisturbed fluid, conservation of angular momentum imposes a zero vorticity constraint on the flow. The lowest-order multipole that conforms to this condition is the potential dipole, which scales as $u \approx U a^{3} r^{-3}$, where $r$ is the radial distance from the translating body by the gradient of the velocity field and the size of the detector, $b$. That is $u^{\prime}=3 U a^{3} b r^{-4}$. There is a fundamental difference in the detection of acoustic signals by planktonic versus benthic organisms; for the latter, which are fixed in space, absolute velocity is relevant.

As pointed out by Yen \& Strickler (1996), the beating appendages of suspension feeding copepods create both an oscillating component and a mean feeding current. The same is true for free-swimming copepods, the mean component being their motion through the fluid. In each case, the mean component is associated with a velocity field that attenuates less rapidly $\left(r^{-1}\right.$ for feeding current and $r^{-2}$ for self-propelled) than the near-field acoustic signal $\left(r^{-4}\right)$. This would suggest that signals associated with vibrations are of minor importance. sitive to the velocity fluctuations associated with a travelling sound wave. Indeed, Lenz \& Hartline (1999) used vibrational stimuli to directly elicit remote escape/attack responses in a calanoid copepod.

The signal transmitted to the surrounding fluid from beating appendages can be modelled as an acoustic dipole (Lighthill 1978, Kalmijn 1988). The strength of the velocity fluctuation attenuates differently in space depending on the distance from the source compared to the acoustic wavelength. Specific formulations are given in Table 3. For an appendage of radial dimension a oscillating at a frequency $\sigma$ (radians $\mathrm{s}^{-1}$ ) with a peak velocity $U$, the amplitude of the velocity fluctuations are given by $u \approx U a^{3} / r^{3}$ for $k r<1$, and $u \approx U k^{2} a^{3} / r$ for $k r>1$, where $k$ is the wave number defined as $k=\sigma / C$, and $c$ is the speed of sound in water. For the marine environment, $c$ lies between 1450 and $1550 \mathrm{~m} \mathrm{~s}^{-1}$.

For the typical frequencies of beating appendages, (10 to $1000 \mathrm{~Hz}$; e.g. Yen \& Strickler 1996), the relatively high speed of sound in seawater means that the wavelength of the associated acoustic wave is 1.5 to $150 \mathrm{~m}$ (Denny 1993), much greater than the distances relevant for individual plankton. Thus for beating appendage detection, the relevant dynamics are described by nearfield acoustics (Eq. a9: Table 3). This has 2 important consequences. Firstly, the attenuation of the signal is nearly always rapid, falling off with an $r^{-3}$ power law with increasing distance from the source. Secondly, because the wavelength is long, the velocity field itself will serve only to translate the detector (copepod) as a whole, yielding no velocity difference between the fluid and the detector. The detectable signal, $u^{\prime}$, is provided

\section{SWIMMING AT HIGH REYNOLDS NUMBER}

While their habitat is generally one of low Reynolds number, occasionally the plankton experience or generate energetic, inertially dominated events. It is these periodic excursions into the high $R e$ (Eulerian) regime that I examine here. The 2 specific examples considered are the generation and detection of the hydromechanical signal associated with the approach of a predatory fish and the rapid escape jump of a copepod.

Hydrodynamic signals generated by high $R e$ motion differ from those at low Re primarily in the volume of fluid influenced by viscosity. At low $R e$, this volume is essentially infinite; at high $R e$, the influence of viscosity is limited to a laminar boundary layer surrounding the moving body. High Re motion is characterised by a strong fore-aft asymmetry. In front of the moving body, the fluid is largely unaffected and is only driven by its own inertia and continuity; behind the moving body, however, vorticity and momentum generated at the body's surface are shed to the bulk of the fluid. Thus, at high $R e$, the hydromechanical signals associated with an approaching or retreating organism differ.

Immediately adjacent to the translating body (Fig. 14), is the so-called laminar boundary layer wherein momentum and vorticity are imparted from the body to the fluid by viscous forces. This boundary layer has a thickness of $O\left(a R e^{-1 / 2}\right)$ and is a region of intense momentum and vorticity transmission to the fluid that is advected aft of the translating body. Within the wake, momentum and vorticity are transmitted to the bulk of the fluid. This can be seen as a zone where the 
fluid relaxes from the disturbance generated by the translating body, and can be modelled as a translating point source of momentum (Batchelor 1967) leading to Eq. (a13) in Table 3. This expression assumes that the along-wake diffusion of momentum is minimal and comparable to the plume generated by a translating point source of a chemical tracer (Csanady 1973, Okubo 1980). Beyond the region where viscosity plays an important role, the flow is essentially inviscid and irrotational in an otherwise undisturbed fluid; i.e. conservative properties of the flow, such as momentum and vorticity, are only transmitted by advection. This type of flow is known as potential flow.

\section{Approaching/attacking fish}

We can model the fluid disturbance in front of a swimming fish as that associated with a translating sphere at high $R e$ where the forward hemisphere approximates the snout of the fish. In front of the laminar boundary layer, the flow field is non-turbulent and described by a potential dipole, given in Eq. (a12) in Table 3.

In a recent study, Viitasalo et al. (1998) demonstrated that the ability of juvenile fish (the stickleback Gasteroseus aculeatus) to successfully approach, attack and capture rheotactic copepod prey was dependent on the final approach speed of the fish. In their study, the rheotactic ability of the prey (in this case Eurytemora affinis) was quantified in a siphon experiment, which showed that the copepod initiated an escape reaction at a flow deformation rate $e^{*}$ of between 0.8 and $9 \mathrm{~s}^{-1}$, with mean at about $2 \mathrm{~s}^{-1}$. This corresponds to a sensitivity $s=1 \mathrm{~mm} \mathrm{~s}^{-1}$ (range 0.4 to $4.5 \mathrm{~mm} \mathrm{~s}^{-1}$ ).

Sticklebacks (approx. length $2 \mathrm{~cm}$, width $0.2 \mathrm{~cm}$ ) cruise at a speed of 1 to $3 \mathrm{~cm} \mathrm{~s}^{-1}$ ( $R e=20$ to 60). Upon visually fixing a prey item at a distance of $2 \mathrm{~cm}$, they approach it by decelerating over a period of 1 to $2 \mathrm{~s}$ to a speed of $0.5 \mathrm{~cm} \mathrm{~s}^{-1}(R e=10)$. The final attack, initiated at a distance of $0.1 \mathrm{~cm}$, is rapid, with speeds of over $6 \mathrm{~cm} \mathrm{~s}^{-1}(R e>120)$. In all instances the Reynolds number exceeds 10, and the thickness of the laminar boundary layer is at most $0.03 \mathrm{~cm}$, i.e. less than the attack distance. Thus, the translating sphere model used by Viitasalo et al. (1998), may be less than ideal in that it is formulated for low Reynolds numbers only.

Directly in front of an approaching fish $(\theta=0)$ the deformation rate of the potential flow is given by:

$$
e(r)=\frac{\partial u_{r}}{\partial r}=3 U \frac{a^{3}}{r^{4}}
$$

which gives a reaction distance:

$$
R=\left(3 U a^{3} / e^{*}\right)^{1 / 2}
$$

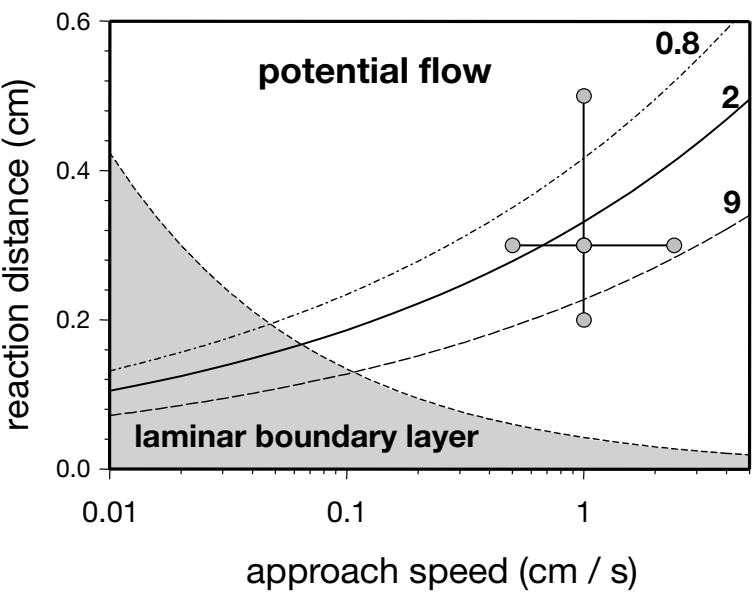

Fig. 15. Reaction distance $R$ of the copepod Eurytemora affinis as a function of the approach speed $U$ of a predatory larval fish (stickleback). Shaded region indicates the laminar boundary layer around the head of the fish where friction is important; in the unshaded region, the flow is dominated by inertia and conforms to a potential flow. The 3 curves indicate estimated $R$ as a function of $U$ based on a potential dipole model of the flow, and the range of threshold deformation rates measured by Viitasalo et al. (1998). Also shown is the range of observations for successful escapes of the copepod also reported by Viitasalo et al. (1998)

Fig. 15 shows calculations of the reaction distance for sticklebacks based on the information in Viitasalo et al. (1998); 3 curves are presented for different values of the threshold deformation rate: $e^{*}=0.8,2$, and $9 \mathrm{~s}^{-1}$ respectively. Also drawn is the curve delineating the laminar boundary layer. Observations of escape events of Eurytemora affinis are plotted as a function of the fish approach speed and reaction distance, and lie reasonably well within the predicted curves. Given the uncertainty of the data, it must be noted that the model used by Viitasalo et al. (1998) also gave a reasonable fit.

\section{Copepod escape jumps}

When alerted to proximate danger, copepods can perform spectacular escape runs, either in the form of high-velocity darts, constant swimming, or a sequence of jumps (Yen \& Strickler 1996). Similar escape responses are exhibited by other zooplankton, e.g. rotifers (Williamson \& Vanderploeg 1988). Escape speeds can reach up to $500 \mathrm{~mm} \mathrm{~s}^{-1}$, corresponding to up to 200 body lengths $\mathrm{s}^{-1}$. That these events have an inherently high Reynolds number (i.e. are inertially dominated) can be seen, for instance, in the results of a study by Yen \& Strickler (1996), who presented a Schlieren image of the hydrodynamic 'footprint' left in the wake of an escaping Euchaeta rimana. This showed mushroom-shaped vortices shed at about $35 \mathrm{~ms}$ intervals 
and with $5 \mathrm{~mm}$ separation. The average Reynolds number for this behaviour is $R e=100$, although it may be considerably higher at the instant that each vortex is shed.

More detailed examinations of the vortex shed by escaping zooplankton have recently been conducted by Gries et al. (1999) for Daphnia pulicaria, and by van Duren et al. 1998 for the copepod Temora longicornis. The latter examines the velocity in the wake of an escape using laser-sheet particle-image velocimetry yielding detailed quantification of the flow field. The hydrodynamics of both the copepod flight and the signal it generates is crucial to the ensuing escape/capture probability. While powerful escape jumps are useful in removing the copepod from immediate risk, they also generate strong signals that can alert the predator to their presence. Indeed, some predator attacks on rheotactic prey appear to be triggered by the prey escape reaction (Yen \& Strickler 1996). Artificial vortices have, in fact, been used in the past to elicit responses in zooplankters, e.g. in examining the matelocating abilities of the copepod T. longicornis (Yen et al. 1998), and the attack response of chaetognaths (Nishii 1998). The moral is clear: if a prey is to escape, it had better be successful, since a half-hearted attempt is worse than no attempt.

A single jump or a rapid swimming bout is initiated when the copepod applies a thrust to the fluid. While more sophisticated models of how copepods generate thrust have appeared (e.g. Morris et al. 1985 considered the motion of pereiopod pairs), for simplicity I assume this to be affected by a single impulse: a constant force $F_{\mathrm{T}}$ applied for a finite time interval, $\Delta t$. During this motion, the copepod accelerates, comes under the influence of drag, and approaches 'terminal' velocity, $U_{0}$. When the copepod stops applying thrust, it decelerates due to drag, and finally comes to a stop. We can model the thrust $F_{\mathrm{T}}$ by considering the rate of change of momentum of fluid pumped by the copepod's swimming appendages. Specifically, if the velocity of pumped fluid relative to the copepod is $u$, and it is pumped through a cross-sectional area of $A$, then in a high Reynolds number regime $F_{\mathrm{T}}=\rho A u^{2}$, where $\rho$ is the density of the fluid. However, there is a maximum rate, $U$, at which the copepod can pump water. This is physically fixed by the rate at which the copepod can move its swimming appendages. Thus, the relative velocity pertinent to the thrust is $u=U-v$, where $v$ is the speed of the copepod through the water. The thrust that can be applied is maximum when the copepod is stationary, decreasing as the copepod accelerates (a similar process occurs when the initial oar strokes move a rowing boat). For simplicity, setting the density of the copepod equal to that of the fluid, and $A=\pi a^{2}$, the equation of motion (from Maxey \& Riley 1982, sum- marised in Eq. a14 to a16 in Table 3) for the copepod can be written as:

$$
\frac{\mathrm{d} v}{\mathrm{~d} t}=\left\{\begin{array}{cc}
\frac{3}{4} \frac{(U-V)^{2}}{a}-\frac{f}{\tau} v & \text { for } t<\Delta t \\
-\frac{f}{\tau} v & \text { for } t \geq \Delta t
\end{array}\right.
$$

Here $v(t)$ is the copepod's velocity through the water and $a$ (in this case) is the equivalent radius of the crosssectional area of the copepod perpendicular to its direction of motion; $\tau=2 a^{2} \rho /(9 \mu)$ is the Stokes time scale, i.e. the time for a particle of radial dimension a to dissipate most of its momentum to the fluid of density $\rho$ and dynamic viscosity $\mu$. For a $100 \mu$ m-radius particle, $\tau$ is approximately $1 \mathrm{~ms}$. The drag factor $f$ is from Clift et al. (1978), reviewed in Fung (1998), and covers the $R e$ range up to $O\left(10^{3}\right)$ :

$$
f=1+0.1315 R e^{\left(0.82-0.05 \log _{10} R e\right)}
$$

Fig. 16 presents a numerical simulation of Eq. (34) subject to Eq. (35) for $a=0.2 \mathrm{~mm}(\tau=5 \mathrm{~ms}), U=500 \mathrm{~mm} \mathrm{~s}^{-1}$, where thrust is applied for $\Delta t=0.01 \mathrm{~s}$. The copepod accelerates rapidly (Fig. 16a), attaining a terminal velocity of $U_{0}=270 \mathrm{~mm} \mathrm{~s}^{-1}$ within $2 \mathrm{~ms}$. The thrust stops at $t=10 \mathrm{~ms}$, and the copepod effectively comes to a halt within the next $20 \mathrm{~ms}$. These parameters roughly correspond to a single power stroke of Euchaeta rimana (Yen \& Strickler 1996). Maximum displacement is a little under $3.2 \mathrm{~mm}$ (Fig. 16b), and most of this is accomplished while the copepod is actively applying thrust. The thrust and drag (Fig. 16c) experienced by the copepod is equal (acceleration $\approx 200 \mathrm{~m} \mathrm{~s}^{-2}$, force $\approx$ $6 \times 10^{-6} \mathrm{~N}$ ) during the constant-speed phase. For the short interval prior to this, thrust is up to 5 times higher. The result is that the fluid experiences a very intense impulse (Fig. 16d) as the copepod initiates its escape. The net force on the fluid falls to zero during the constant-speed phase, and then reverses when the thrust is turned off and only drag is active. The main features of the motion are similar to the more sophisticated model (Morris et al. 1985) for Pleuromamma xiphias (length $6 \mathrm{~mm}$ ), which gave results (maximum thrust $25 \times 10^{-6} \mathrm{~N}, \Delta t=15 \mathrm{~ms}, U_{0}=300 \mathrm{~mm} \mathrm{~s}^{-1}$, and distance travelled $=3.8 \mathrm{~mm}$ ).

If the initial momentum impulse applied to the fluid (Fig. 16d) is sufficiently strong, a vortex will evolve, propagating behind the copepod and entraining fluid as it moves. Theoretical considerations (Maxworthy 1972, Shariff \& Leonard 1992) suggest that the temporal evolution of the translational velocity, $V$, of the vortex is of the form:

$$
V=A\left(t+t_{0}\right)^{-1}
$$

where $t_{0}$ is a 'virtual' time origin, and $A$ is a constant. Unfortunately, the values of $A$ and $t_{0}$ cannot be 
derived from first principles, and must be empirically determined. As an example, the data of van Duren (2000) on the vortex generated by a Temora longicornis Copedodite Stage I (length $330 \mu \mathrm{m}$ ) escape reaction yields a reasonable fit for $A=2$ $\mathrm{mm}$ and $t_{0}=0.1 \mathrm{~s}$. The dimension of the vortex can be estimated from conservation of momentum. Thus, if $b$ is the radial dimension of the vortex, $V b^{3} \approx$ constant. Defining $X$ as the distance of the vortex from the point of impulse, then:

$$
\begin{aligned}
& X=A \ln \left(1+t / t_{0}\right) \\
& V=V_{0} \exp (-X / A) \\
& b=b_{0} \exp (X /(3 A))
\end{aligned}
$$

where $V_{0}=A / t_{0}$ is the initial velocity (at $t=0$ ) and $b_{0}$ is the initial radial dimension of the vortex. It is reasonable to assume that $b_{0}$ scales with the size of the organism generating the vortex.

By equating the initial momen-
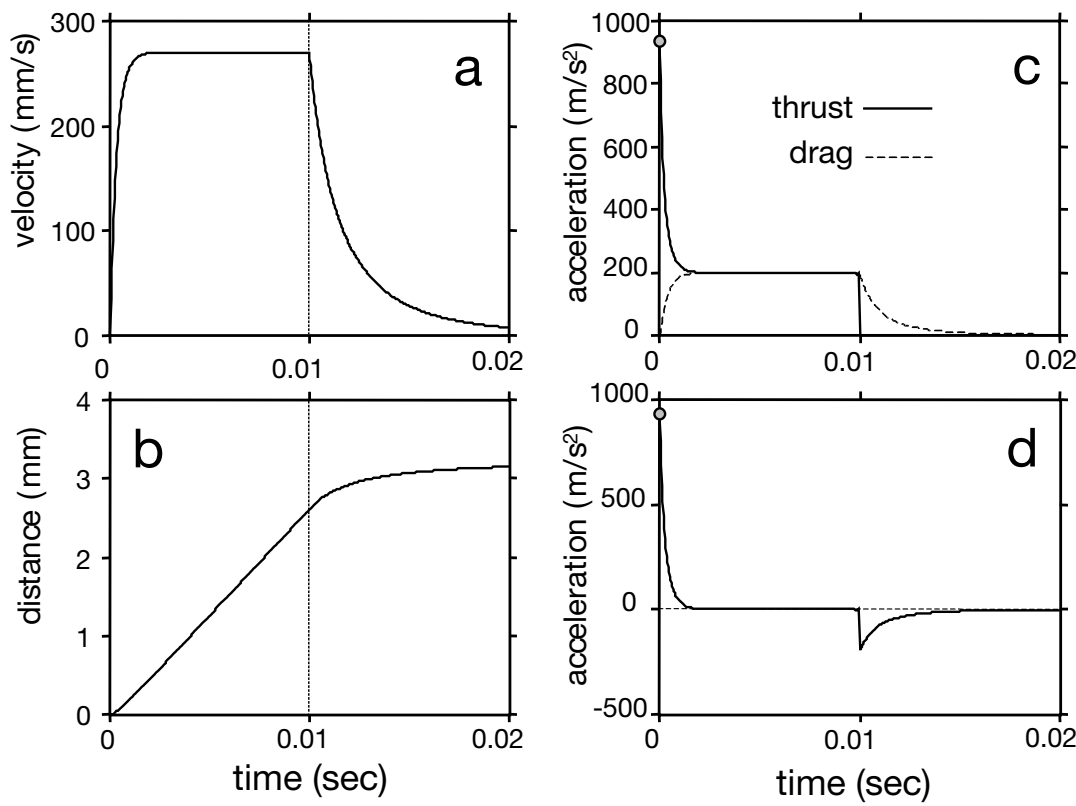

Fig. 16. Simulation of the power stroke of a copepod escape jump. The copepod has an effective radius of $0.2 \mathrm{~mm}$, and a maximum 'pumping' velocity of $500 \mathrm{~mm}$ $\mathrm{s}^{-1}$. Thrust is applied for $10 \mathrm{~ms}$. (a) Velocity; (b) distance (c) and (d) acceleration (force per unit mass) experienced by the copepod (thrust and drag) and by the fluid respectively tum imparted by the copepod to the fluid with the initial conditions of the vortex, 1 can approximate:

$$
\int_{0}^{\Delta t}\left(F_{\mathrm{T}}-F_{\mathrm{D}}\right) \mathrm{d} t \approx \pi \rho U^{2} a^{2} \tau / 2 \approx 4 / 3 \pi \rho V_{0} b_{0}{ }^{3}
$$

Thus, by combining the motion of the copepod (Eqs 34 \& 35) and the motion of the vortex (Eqs 37 to 39) one has a potential means of predicting the escape success as a balance between the ability to flee danger (jump length), and the exposure to attack risk (hydromechanical signal of the vortex). Unfortunately, knowledge of the small vortices at the scale commensurate with copepod escape jumps is still too incomplete to make quantitative predictions at this time.

A few salient points, however, can be drawn from a scale analysis of the processes involved. The time scale associated with small vortices is long compared to typical time scales for copepod jumps (swimming interval $\Delta t+$ Stokes time scale $\tau$ ). Even for the relatively weak vortex generated by Temora longicornis CI reported by van Duren (2000), velocities in excess of $1 \mathrm{~mm} \mathrm{~s}^{-1}$ were evident $1 \mathrm{~s}$ after the impulse event. The Stokes time scale for $T$. longicornis $\mathrm{CI}$ is in the order of $1 \mathrm{~ms}$, and the escape interval in the order of $10 \mathrm{~ms}$. In this time interval, the vortex is barely diminished and still has $90 \%$ of its initial velocity. Thus, a predatory copepod with sensitivity of $s=100 \mu \mathrm{m} \mathrm{s}^{-1}$ can sense the vortex (equating $V$ with $s$ in Eq. 36) up to $10 \mathrm{~mm}$ from its origin for $20 \mathrm{~s}$ after the jump event, and over a volume

with radius of $2 \mathrm{~mm}$. This analysis poses the question: will not a large powerful eddy confuse the predator rather than alert it to a potential prey? This may very well be the case for a relatively large prey generating a correspondingly large vortex; however, small-scale vortices remain coherent over quite a long distance. Again taking T. longicornis CI as an example, the size of the vortex increases by $<20 \%$ over a travel distance of $1 \mathrm{~mm}$, and doubles in size only after $>4 \mathrm{~mm}$ travel distance. Thus, not only does the vortex carry information about the size of its generating organism over relatively long distances, but the coherence of the vortex suggests that directional information is potentially available to the predator (Fig. 17). Seen in this way, an escape jump for T. longicornis CI is a high-risk manoeuvre.

The risk factor involved in generating a conspicuous vortex can be measured in terms of the initial momentum imparted to the fluid. Assuming that the vortex initially scales with $a$, the risk is proportional to $U^{2} a / \mu$ from Eq. (40). In comparison, the benefit derived from the escape bout (i.e. distance) depends primarily on the swimming duration $\Delta t$ and its terminal velocity $U_{0}$. Examining the terminal velocity (solving $\mathrm{d} v / \mathrm{d} t=0$ for $t<t_{0}$ in Eq. 32), we find $U_{0}=U /\left(1+(12 f / R e)^{1 / 2}\right)$ which gives $U_{0} \propto a^{1 / 2}$ for $R e>1$. Thus, with respect to size, risk increases faster than benefit ( $a$ vs $a^{1 / 2}$ ) with increasing size. However, other factors certainly play a role. Escape distance also depends on escape duration $\Delta t$, 


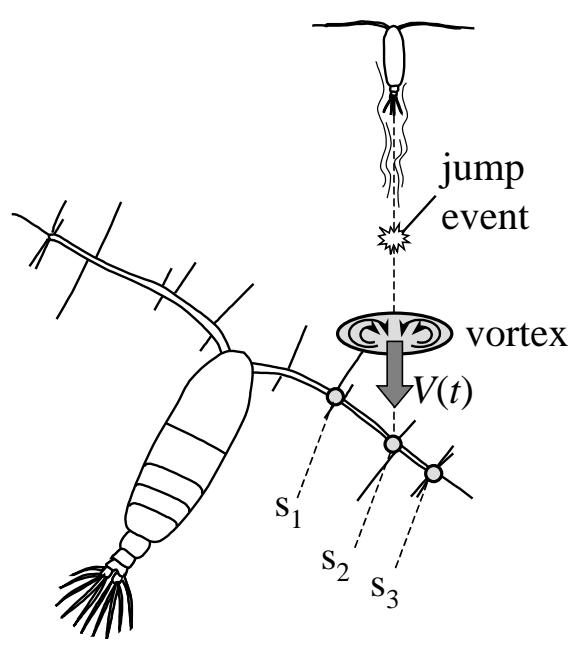

Fig. 17. Vortex detection. A predatory copepod can potentially gain higher-order information from the detection of a vortex shed from an escape reaction of, for instance a nauplius. On the planktonic scale, the size and velocity of the vortex changes only slowly over time and distance travelled. Furthermore, high $R e$ flows can maintain sharp velocity gradients. Thus, the measured size and velocity of the vortex relates to the jump speed and size of the escaping copepod. The timing of the signal as it passes different setae, $\mathbf{s}_{1}, \mathrm{~s}_{2}$ and $\mathrm{s}_{3}$, gives directional information, indicating the path of the escaping nauplius

and the maximum fluid speed, $U$, both of which are expected to be strongly size-dependent.

\section{TURBULENCE AND SIGNAL DETECTION}

Under natural conditions, organisms experience not only the hydromechanical signals generated by other organisms, but also a myriad of signals associated with the physical forcing of the environment. On the planktonic scale, these signals are within the turbulentenergy spectrum, close to (and often spanning) the transition from the inertial sub-range to the dissipative sub-range. This transition occurs at the Kolmogorov scale, $L_{\mathrm{K}}=\left(v^{3} / \varepsilon\right)^{1 / 4}$ where $v$ is the kinematic viscosity of water. Turbulence has been proposed to constitute an important environmental factor for plankton, particularly regarding its role in enhancing planktonic encounter rates (Eq. 1 and Rothschild \& Osborn 1988, Evans 1989). These positive aspect of turbulence have been demonstrated for copepods in laboratory studies (e.g. Marrasé et al. 1990, Saiz et al. 1992). On the other hand, it has been proposed that turbulence can interfere with the detection of hydromechanical signals (Kiørboe \& Saiz 1995), thus reducing the effective detection distance for particular motility classes of organisms. As is often the case in environmental pro- cesses, turbulence effects a dome-shaped response (MacKenzie et al. 1994). Indeed, the few field measurements available indicate that negative aspects of turbulence often dominate in nature (Irigoien et al. 2000, Visser et al. 2001).

In order to examine turbulent interference with signal detection, consider a copepod with inherent sensitivity, $s_{0}$, detecting a swimming ciliate at low $R e$. The ciliate has a radial dimension, $a$, and swims with velocity $U$. As outlined earlier ('Stokes-flow solutions': 3rd subsection), the velocity scale associated with swimming is $u \propto U(a / r)^{2}$, and the detection distance is:

$$
R(0) \propto a\left(U / s_{0}\right)^{1 / 2}
$$

The effect of turbulence is to introduce velocity differences between the body of the copepod and the setae positioned along its antennae. Taking the length of each antenna to be $b$, the magnitude of the turbulent velocity at the antenna tips is given by:

$$
u_{t}=\alpha(\varepsilon b)^{1 / 2}
$$

where $\varepsilon$ is the turbulent dissipation rate, and $\alpha$ is Richardson's constant $(\cong 1)$. This describes the case where $b$ is $>$ Kolmogorov scale. For detection, we can assume that the ratio of the signal (i.e. from a swimming ciliate) to noise (turbulence) must exceed a constant, $\kappa$. From this, we get:

$$
R(\varepsilon) \propto\left\{\frac{U a^{2}}{\alpha \kappa(\varepsilon b)^{1 / 3}}\right\}^{1 / 2} \propto \varepsilon^{-1 / 6}
$$

i.e., detection distance decreases with increasing turbulence. $\mathrm{A}^{-1 / 6}$ power law is not in itself a very strong dependence. However, considering the natural range of turbulence ( $\varepsilon$ from $10^{-6}$ to $1 \mathrm{~cm}^{2} \mathrm{~s}^{-3}$; e.g. Estrada \& Berdalet 1997), turbulent interference with signal detection can bring about an order of magnitude change in reaction distance. Saiz \& Kiørboe (1995) published results from a laboratory study of Acartia tonsa feeding on the ciliate Strombidium sulcatum under varying degrees of turbulence. From the observed clearance rate, the inferred detection distance was deduced form the associated encounter-rate equation (similar to Eq. 1 of the present paper). These results are plotted in Fig. 18, and show remarkably good agreement with the $\varepsilon^{-1 / 6}$ scaling relationship postulated above.

While this analysis demonstrates the role of turbulence in impairing remote detection of hydromechanical signals, there are a few important riders. Firstly, the scaling law that arises from this example is dependent on the turbulent regime $\left(L_{\mathrm{K}}<\right.$ length scale of detector) and applies to a self-propelled organism (stresslet flow model). These conditions are consistent with the laboratory experiment of Saiz \& Kiørboe (1995). Smaller de- 
tector-length scales (compared to $L_{\mathrm{K}}$ ), and different motility modes will be susceptible to turbulence to a greater or lesser degree. Secondly, biological factors also play a role, from switching of feeding behaviour (e.g. Costello et al. 1990, Marrasé et al. 1990) to the bioenergetics of living in turbulent conditions (e.g. Saiz et al. 1992).

\section{CONCLUSIONS}

The purpose of this work has been largely to summarise and review the physics associated with hydromechanical signals that can potentially be generated and detected by zooplankton. A summary of the flow perturbations arising from different classes of motion is provided in Table 3, and it is hoped that this summary will become a useful tool for plankton researchers in the future. The summary, however, does not claim to be exhaustive. There is a large class of processes, particularly in the inertial-turbulent range, which are poorly understood and which can have important influences on how plankton perceive and interact with their environment. An important case in point is the production of vortices by fleeing copepods ('Swimming at high Reynolds number'; Copepod escape jumps). Moreover, the treatment here is highly idealised. While some plankton are essentially spherical in shape, others are not. This is certainly the case for copepods. Thus, drag and other body-form forces such as lift can certainly be different than for a sphere. Likewise thrust is applied not as a point force but distributed over a volume of space. While detailed observations (e.g. by van Duren 2000) and advanced computer models (e.g. by Jiang et al. 1999) are currently addressing these factors, it remains difficult to say what the overall effect is. It is only possible to make a few quantitative comparisons of the theoretical analysis with actual observations. In less well-documented cases, examples using realistic parameters have demonstrated the underlying mechanisms and feasibility of the remote detection of hydromechanical signals in the plankton.

An important part of this work is to advance the paradigm that different modes of plankton motility and behaviour generate different classes of hydromechanical signals that attenuate differently in space. That is, the hydromechanical signal generated by a self-propelled organism is different from that generated by a feeding current, that is different again to that generated by a passive particle entrained in a deforming flow. Given that detection is triggered when the velocity between the detector and fluid exceeds some threshold, scaling laws can be constructed for hydromechanical detection in the plankton as a function of motility modes. These results are summarised in Table 4, which summarizes the 2 sets of detection dis-

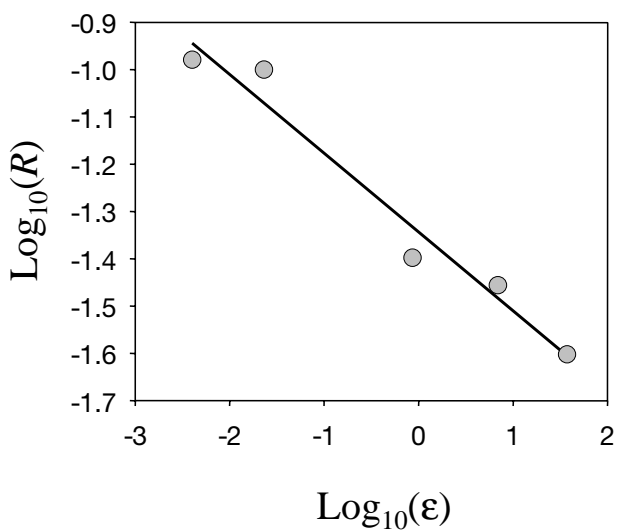

Fig. 18. Acartia tonsa. Estimated reaction distance, $R(\mathrm{~cm})$, as a function of dissipation rate, $\varepsilon\left(\mathrm{cm}^{2} \mathrm{~s}^{-3}\right)$, for the copepod feeding on the ciliate Stromidium sulcatum in a laboratory study (Kiørboe \& Saiz 1995). Reaction distance estimated from observed clearance rates, $\beta_{\mathrm{o}}$ and by numerically solving the encounter equation $\beta_{0}=\pi R^{2}\left(U^{2}+2 \alpha^{2}(\varepsilon R)^{2 / 3}\right)^{1 / 2}$. The Kolmogorov scale ranges from about $1 \mathrm{~mm}$ to $100 \mu \mathrm{m}$, which is $\leq$ the length scale of $A$. tonsa (antenna span, $2 b=2 \mathrm{~mm}$ )

tance scaling relations as a function of the relative size of the detector $(b)$ and the detected organism $(a)$. When the organism generating the hydromechanical signal is much smaller than that detecting it $(a \ll b)$, only the absolute velocity disturbance need be considered. However, when the organism generating the disturbance becomes comparable or larger in size that the detector $(a \geq b)$ the motion of the detector as a whole becomes important. In this case, the pertinent signal strength becomes the product of the gradient of the velocity (more properly the deformation rate) and the detector length scale.

Table 4. Summary of the detection distance scaling for different motility modes. $U$ is the speed of the translating body, and $a$ is its radial dimension. Detector length scale is $b$, and detection is triggered when the velocity difference between the detector and fluid exceeds the sensitivity threshold $s$

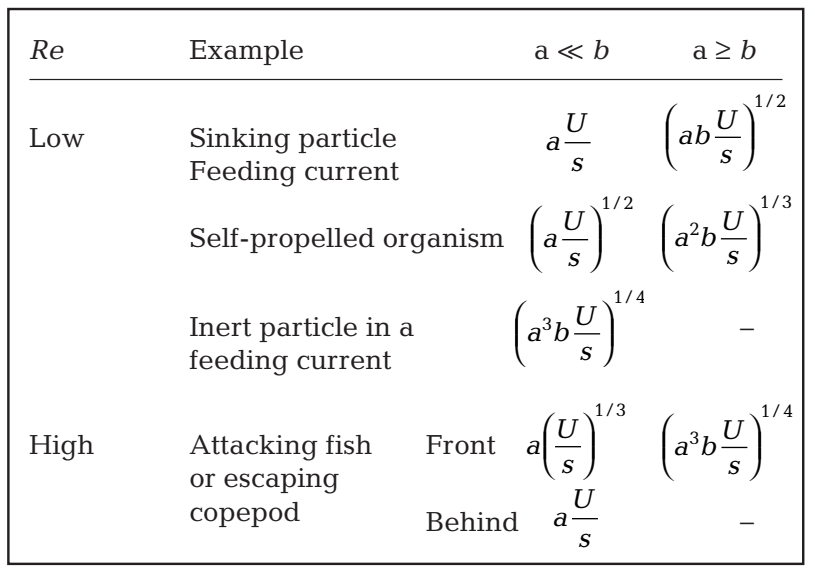


The models considered here are idealised not only as regards hydrodynamics but also in regard to the biological aspects of signal detection and response. The rationale of concentrating on the signal strength of a single detector highlights the feasibility of using hydromechanical signals for remote detection. However, a wide range of biological factors also play important roles. These range from how signals are detected (e.g. setal bending and enervation, susceptibility to other components of the signal such as displacement, acceleration, and chemical stimuli) through signal processing (e.g. timing and spatial separation of multiple stimuli) to behavioural response (e.g. habituation to stimuli, motivational state of predator and prey). For instance, simply detecting a signal falls far short of using the information content of the signal to locate a potential prey, predator or mate. For this, multiple detectors should be considered. Copepods often have a whole array of detectors projecting into the surrounding fluid, principally the first antennae and the array of setae spanning them. Indeed, a spatial array of sensors is found in many species, e.g. chaetognaths have mechanoreceptors positioned along the whole length of their bodies. An earlier section ('Stokes flow solutions'; Directional information) briefly considers a simple case for a copepod locating a small swimming organism. Key to this is that copepod setae appear to be sensitive to the direction of velocity disturbances. Furthermore, a higher degree of information can potentially be gathered by the position, number and timing of setal excitation in discerning the location, size and velocity of the organism from which the hydromechanical signal emanates.

Finally, the idealised nature of the models presented here (spherical body shape, simple force distribution, and single detector), while far from perfect, yields a transparent and quantifiable prediction of the detection scale associated with hydromechanical signals in the plankton. This is achieved with a minimum number of parameters: body size (of both generator and detector), speed, and detector sensitivity. Using this analysis and known parameter values, the feasibility of detection has been demonstrated for copepods detecting other copepods' feeding currents, swimming ciliates and rotifers as well as approaching fish larvae. These examples are well supported by observations. Less well-documented is the detection of inert particles entrained into a feeding current. The analysis shows that in principle this is feasible, suggesting that in addition to being a means of transporting food towards its mouth parts, a copepod's feeding current can also act as part of its remote detection system. In a similar manner, an inert particle can in principle be detected by a free-swimming copepod through a measurable perturbation of the copepod's own flow field. Although not specifically explored here, the same process can be expected to be active for a sinking copepod passing a non-sinking particle. Thus, even planktonic organisms that are totally inactive (do not swim, sink or generate a feeding current) can still be detected hydromechanically by copepods.

Acknowledgements. I would like to thank Thomas Kiørboe, Peter Tiselius, Markku Viitasalo and George Jackson for their interest, encouragement and comments in preparing this manuscript. I would also like to thank Hiroaki Saito for his comments and for translating some Japanese text for me. This study was part of PROVESS (Processes Of Vertical Exchange in Shelf Seas), an EU-funded project under the MAST III programme (contract number MAS3-CT97-0159).

\section{LITERATURE CITED}

Batchelor GK (1967) An introduction to fluid dynamics. Cambridge University Press, Cambridge

Berg HC (1993) Random walks in biology. Princeton University Press, Princeton, NJ

Bundy MH, Paffenhöfer GA (1993) Innervation of copepod antennules investigated using laser scanning confocal microscopy. Mar Ecol Prog Ser 102:1-14

Bundy MH, Gross TF, Vanderploeg HA, Strickler JR (1998) Perception of inert particles by calanoid copepods: behavioral observations and a numerical model. J Plankton Res 20:2129-2152

Buskey EJ (1984) Swimming pattern as an indicator of the roles of copepod sensory systems in the recognition of food. Mar Biol 79:165-175

Caparroy P, Pérez MT, Carlotti F (1998) Feeding behaviour of Centropages typicus in calm and turbulent conditions. Mar Ecol Prog Ser 168:109-118

Childress S (1981) Mechanics of swimming and flying. Cambridge University Press, Cambridge

Childress S, Koehl MAR, Miksis M (1987) Scanning currents in Stokes flow and the efficient feeding of small organisms. J Fluid Mech 177:407-426

Clift R, Grace JR, Webber ME (1978) Bubbles, drops and particles. Academic Press, San Diego, CA

Costello JH, Strickler JR, Marrasé C, Trager G, Zeller R, Freise AJ (1990) Grazing in a turbulent environment: behavioural response of the calanoid copepod Centropages hamatus. Proc Natl Acad Sci USA 87:1648-1652

Costello JH, Loftus R, Waggett R (1999) Influence of prey detection on capture sucess for the ctenophore Mnemiopsis leidyi feeding upon adult Acartia tonsa and Oithona colcarva copepods. Mar Ecol Prog Ser 191:207-216

Csanady GT (1973) Turbulent diffusion in the environment. D. Reidel, Dordrecht

DeMott WR, Watson MD (1991) Remote detection of algae by copepods: response to algal size, odors and motility. J Plankton Res 13:1203-1222

Denny MW (1993) Air and water: the biology and physics of life's media. Princeton University Press, Princeton, NJ

Doall MH, Colin SP, Yen J, Strickler JR (1998) Locating a mate in 3D: the case of Temora longicornis. Philos Trans R Soc Lond Biol Sci 353:681-687

Emlet RB, Strathman RR (1985) Gravity, drag, and feeding currents of small zooplankton. Science 228:1016-1017

Estrada M, Berdalet E (1997) Phytoplankton in a turbulent world. Sci Mar 61:125-140 
Evans GT (1989) The encounter speed of moving predator and prey. J Plankton Res 11:415-417

Feigenbaum D, Reeve MR (1977) Prey detection in the Chaetognatha: response to a vibrating probe and experimental determination of attack distance in large aquaria. Limnol Oceanogr 22:1052-1058

Fenchel T, Jonsson PR (1988) The functional biology of Strombidium sulcatum, a marine oligotrich ciliate (Ciliophora, Oligotrichina). Mar Ecol Prog Ser 48:1-15

Fields DM, Yen J (1997a) Implications of the feeding current structure of Euchaeta rimana, a carnivorous pelagic copepod, on the spatial orientation of their prey. J Plankton Res 19:79-95

Fields DM, Yen J (1997b) The escape behaviour of marine copepods in response to a quantifiable fluid mechanical distrurbance. J Plankton Res 19:1289-1304

Fung JCH (1998) The effect of nonlinear drag on the settling velocity of particles in homogeneous isotropic turbulence. J Geophys Res 103:27905-27917

Gallager SM (1993) Hydrodynamic disturbances produced by small zooplqankton: case study for the veliger larva of a bivalve mollusc. J Plankton Res 15:1277-1296

Gilbert JJ (1994) Jumping behavior in the oligotrich ciliates Strombilium velox and Halteria grandinella, and its significance as a defense against rotifer predators. Microb Ecol $27: 189-200$

Gill CW (1985) The response of a restrained copepod to tactile stimulation. Mar Ecol Prog Ser 21:121-125

Gill CW, Poulet SA (1988) Impedance traces of copepod appendage movements illustrating sensory feeding behaviour. Hydrobiologia 167/168:303-310

González HE, Smetacek V (1994) The possible role of the cyclopoid copepod Oithona in retarding vertical flux of zooplankton faecal material. Mar Ecol Prog Ser 113:233-246

Gries T, Jöhnk K, Fields DM, Strickler JR (1999) Size and structure of 'footprints' produced by Daphnia: impact of animal size and density gradient. J Plankton Res 21:509-523

Haury LR, Kenyon DE, Brooks JR (1980) Experimental evaluation of the avoidance reaction of Calanus finmarchicus. J Plankton Res 2:187-202

Hwang JS, Costello JH, Strickler JR (2000) Copepod grazing in turbulent flow: elevated foraging behaviour and habituation of escape responses. J Plankton Res 16:421-431

Irigoien X, Harris RP, Head RN (2000) Does turbulence play a role in feeding and reproduction of Calanus finmarchicus. J Plankton Res 22:399-407

Jack JD, Gilbert JJ (1993) Susceptibilities of different-sized ciliates to direct suppression by small and large cladocerans. Freshw Biol 29:19-29

Jackson GA (1990) A model of the formation of marine algal flocs by physical coagualtion processes. Deep-Sea Res 37: $1197-1211$

Jackson GA (1993) Flux feeding as a possible mechanism for zooplankton grazing and its implications for vertical flux. Limnol Oceanogr 38:1328-1331

Jakobsen HH (2001) Escape response of planktonic protists to fluid mechanical signals. Mar Ecol Prog Ser 214:67-78

Jiang H, Meneveau C, Osborn TR (1999) Numerical study of the feeding current around a copepod. J Plankton Res 21: 1391-1421

Jonsson PR, Tiselius P (1990) Feeding behaviour, prey detection and capture efficiency of the copepod Acartia tonsa feeding on planktonic ciliates. Mar Ecol Prog Ser 60:35-44

Jonsson PR, André C, Lindegarth M (1991) Swimming behaviour of marine bivalve larvae in a flume boundary-layer flow: evidence for near-bottom confinement. Mar Ecol Prog Ser 79:67-76
Kalmijn AJ (1988) Hydrodynamic and acoustic field detection. In: Atema J, Fay RR, Popper AN, Tavolga WN (eds) Sensory biology of aquatic organisms. Springer-Verlag, New York, p 83-130

Kamykowski D, Reed RE, Kirkpatrick GJ (1992) Comparison of sinking velocity, swimming velocity, rotation and path characteristics among six marine dinoflagellates. Mar Biol 113:319-328

Kerfoot WC (1978) Combat between predatory copepods and their prey: Cyclops, Epischura and Bosmina. Limnol Oceanogr 23:1102

Kessler JO (1986) The external dynamics of swimming microorgainisms. Prog Phycol Res 4:258-307

Kim S, Karrila SJ (1991) Microhydrodynamics. ButterworthHeinemann, Boston, MA

Kiørboe T, Saiz E (1995) Planktivorous feeding in calm and turbulent environments, with emphasis on copepods. Mar Ecol Prog Ser 122:135-145

Kiørboe T, Visser AW (1999) Predator and prey perception in copepods due to hydromechanical signals. Mar Ecol Prog Ser 179:81-95

Kiørboe T, Saiz E, Visser AW (1999) Hydrodynamic signal perception in the copepod Acartia tonsa. Mar Ecol Prog Ser 179:97-111

Kirk KL (1985) Water flows produced by Daphnia and Diaptomus: implications for prey selection by mechanosensory predators. Limnol Oceanogr 30:679-686

Kirk KL, Gilbert JJ (1988) Escape behavior of Polyarthra in response to artificial flow stimuli. Bull Mar Sci 43:551-560

Koehl MAR, Strickler JR (1981) Copepod feeding currents: food capture at low Reynolds number. Limnol Oceanogr 26:1062-1073

Landry MR (1980) Detection of prey by Calanus pacificus: implications of the first antennae. Limnol Oceanogr 25: 545-549

Leal LG (1992) Laminar flow and convective transport processes. Butterworth-Heinemann, Boston, MA

Legier-Visser MF, Mitchell JG, Okubo A, Fuhrman JA (1986) Mechanoreception in calanoid copepods. Mar Biol 90: 529-535

Lenz PH, Hartline DK (1999) Reaction times and force production during escape behavior of a calanoid copepod, Undinula vulgaris. Mar Biol 133:249-258

Lenz PH, Yen J (1993) Distal setal mechanoreception of the first antennae of marine copepods. Bull Mar Sci 53:170-179

Lenz PH, Weatherby TM, Weber W, Wong KK (1996) Sensory specialization along the first antenna of a calanoid copepod, Pleuromamma xiphias (Crustacea). Mar Freshw Behav Physiol 27:221

Lighthill J (1975) Mathematical biofluid dynamics. Society for Industrial and Applied Mathematics, Philadelphia, PA

Lighthill J (1978) Waves in fluids. Cambridge University Press, Cambridge

MacKenzie BR, Miller TJ, Cyr S, Leggett WC (1994) Evidence for a dome-shaped relationship between turbulence and larval fish ingestion rates. Limnol Oceanogr 39:1790-1799

Marrasé C, Costello JH, Granata TC, Strickler JR (1990) Grazing in a turbulent environment. II. Energy dissipation, encounter rates and efficacy of feeding currents in Centropages hamatus. Proc Natl Acad Sci USA 87:1653-1657

Maxey MR, Riley JJ (1982) Equation of motion for a small rigid sphere in a nonuniform flow. Phys Fluids 26:883-889

Maxworthy T (1972) The structure and stability of vortex rings. J Fluid Mech 51:15-32

Montgomery JC, MacDonald JA (1987) Sensory tuning of lateral line receptors in aquatic fish to movements of planktonic prey. Science 235:195-196 
Morris MJ, Gust G, Torres JJ (1985) Propulsion efficiency and cost of transport for copepods: a hydromechanical model of crustacean swimming. Mar Biol 86:283-295

Newbury TK (1972) Vibrational perception by chaetognaths. Nature 236:459-460

Nishii S (1998) Hydromechanical perception by chaetognaths. MSc thesis. Mie University, Tsu-shi, Japan

Okubo A (1980) Diffusion and ecological problems: mathematical models. Springer-Verlag, Berlin

Paffenhöfer GA, Strickler JR, Alcaraz M (1982) Suspensionfeeding by herbivorous calanoid copepods: a cinematographic study. Mar Biol 67:193-199

Rothschild BJ, Osborn TR (1988) Small-scale turbulence and plankton contact rates. J Plankton Res 10:465-474

Saiz E, Kiørboe T (1995) Predatory and suspension feeding of the copepod Acartia tonsa in turbulent environments. Mar Ecol Prog Ser 122:147-158

Saiz E, Alcaraz M, Paffenhöfer GA (1992) Effects of smallscale turbulence on feeding rate and gross-growth efficiency of three Acartia species (Copepoda: Calanoida). J Plankton Res 14:1085-1097

Shariff K, Leonard A (1992) Vortex rings. Annu Rev Fluid Mech 24:235-279

Singarajah KV (1969) Escape reactions of zooplankton: the avoidance of a pursuing siphon tube. J Exp Mar Biol Ecol 3:171-178

Strickler JR (1982) Calanoid copepods, feeding currents and the role of gravity. Science 218:158-160

Strickler JR (1985) Feeding currents in calanoid copepods: two new hypotheses. Symp Soc Exp Biol 89:459-485

Strickler JR, Bal AL (1973) Setae of the first antennae of the copepod Cyclops scutifer (Sars): their structure and importance. Proc Natl Acad Sci USA 70:2656-2659

Svensen C, Kiørboe T (2000) Remote prey detection in Othona similis: hydromechanical versus chemical cues. J Plankton Res 22:1155-1166

Tiselius P, Jonsson PR (1990) Foraging behaviour of six calanoid copepods: observations and hydrodynamic analysis. Mar Ecol Prog Ser 66:23-33

Tiselius P, Jonsson PR, Kaartveldt S, Olsen EM, Jørstad T (1997) Effects of copepod foraging behavior on predation risk: an experimental study of the predatory copepod Parechaeta norvegica feeding on Acartia clausi and A.tonsa (Copepoda). Limnol Oceanogr 42:164-170

Vanderploeg HA, Paffenhöfer GA, Leibig JR (1990) Concentration-variable interactions between calanoid copepods and particles of different food quality: observations and hypotheses. In: Hughes RN (ed) Behavioural mechanisms

Editorial responsibility: Otto Kinne (Editor),

Oldendorf/Luhe, Germany for food selection. Springer, Heidelberg, p 595-613

van Duren LA (2000) Moving (in) water. Behavioural kinematics, energetics and hydrodynamics of the calanoid copepod Temora longicornis (Müller). PhD thesis, University of Groningen

van Duren LA, Stamhuis EJ, Videler JJ (1998) Reading the copepod personal ads: increasing encounter probability with hydromechanical signals. Phil Trans R Soc Lond Biol Sci 353:691-700

Viitasalo M, Kiørboe T, Flinkman J, Pedersen LW, Visser AW (1998) Predation vulnerability of planktonic copepods: consequences of predator foraging strategies and prey sensory abilities. Mar Ecol Prog Ser 175:129-142

Visser AW, Jonsson PR (2000) On the reorientation of nonspherical prey particles in a feeding current. J Plankton Res 22:761-777

Visser AW, Saito H, Saiz E, Kiørboe T (2001) Observations of copepod feeding and vertical distribution under natural turbulent condirtions in the North Sea. Mar Biol 138:1011-1019

Vogel S (1994) Life in moving fluids: the physical biology of flow. Princeton University Press, Princeton

Williamson CE (1987) Predator-prey interactions between omnivorous diaptomid copepods and rotifers: the role of prey morphology and behavior. Limnol Oceanogr 32:167-177

Williamson CE, Vanderploeg HA (1988) Predatory suspension-feeding in Diaptomus: prey defenses and the avoidance of cannabalism. Bull Mar Sci 43:561-572

Yen J (2000) Life in transition: balancing inertial and viscous forces by planktonic copepods. Biol Bull (Woods Hole) 198:213-224

Yen J, Strickler JR (1996) Advertisement and concealment in the plankton: what makes a copepod hydrodynamically conspicuous? Invertebr Biol 115:191-205

Yen J, Sanderson BG, Strickler JR, Okubo A (1991) Feeding currents and energy dissipation by Euchaeta rimana, a subtropical pelagic copepod. Limnol Oceanogr 36:362-369

Yen J, Lenz PH, Gassie DV, Hartline DK (1992) Mechanoreception in marine copepods: electrophysiological studies on the first antennae. J Plankton Res 14:495-512

Yen J, Weissburg MJ, Doall MH (1998) The fluid physics of signal perception by mate-tracking copepods. Phil Trans R Soc Lond Biol Sci 353:787-804

Zaret RE (1980) The animal and its viscous environment. In: Kerfoot WC (ed) Evolution and ecology of zooplankton communities. University Press of New England, Hanover, p 3-9

Submitted: October 26, 2000; Accepted: February 21, 2001

Proofs received from author(s): October 26, 2001 